\title{
Phospholipid Composition of APOE Lipoproteins Affects Microglia Activation in an Isoform-specific Manner
}

\section{Nicholas Fitz}

University of Pittsburgh

KyongNyon Nam

University of Pittsburgh

Cody Wolfe

University of Pittsburgh https://orcid.org/0000-0003-2874-4944

\section{Florent Letronne}

University of Pittsburgh

\section{Brittany Playso}

University of Pittsburgh

\section{Bistra lordanova}

University of Pittsburgh https://orcid.org/0000-0001-7015-5006

\section{Takashi Kozai}

University of Pittsburgh

\section{Richard Biedrzycki}

University of Pittsburgh

\section{Valerian Kagan}

University of Pittsburgh https://orcid.org/0000-0002-7245-1885

\section{Yulia Tyurina}

University of Pittsburgh

\section{Xianlin Han}

The University of Texas Health Science Center at San Antonio https://orcid.org/0000-0002-8615-2413 Iliya Lefterov

university of Pittsburgh

\section{Rada Koldamova ( $\square$ radak@pitt.edu )}

University of Pittsburgh https://orcid.org/0000-0002-6761-0984

\section{Article}

Keywords: APOE, Transcriptomics, Microglia, Alzheimer's disease, Amyloid beta, Neurodegeneration, Trem2, RNA-sequencing, Single cell, Infusion 
Posted Date: October 16th, 2020

DOl: https://doi.org/10.21203/rs.3.rs-83085/v1

License: (c) (1) This work is licensed under a Creative Commons Attribution 4.0 International License. Read Full License

Version of Record: A version of this preprint was published at Nature Communications on June 7th, 2021. See the published version at https://doi.org/10.1038/s41467-021-23762-0. 


\section{Abstract}

Apolipoprotein E4 (APOE) is the strongest genetic risk factor for Alzheimer's disease (AD). Our lipidomic analysis identified a common phospholipid signature with a high level of correlation between APOEع3/3 and APOE\&4/4 AD postmortem brain samples and native lipoproteins isolated from astrocyte conditioned media of mice expressing human APOE3 or APOE4. Behavioral testing demonstrated that native $E 3$ lipoproteins were more effective than $E 4$ at ameliorating the harmful effects of $A \beta$ on cognition. We posit that APOE isoform-specific differences in the phospholipid composition of native lipoproteins prompt a differential microglial response. Using time-lapse in vivo two-photon imaging we compared the effect of E3 or E4 infused with $A \beta$ and determined that $E 3$ lipoproteins induced a faster microglial migration towards $A \beta$. To determine how E3 and E4 lipoproteins affect microglial transcriptome in response to $A \beta$ we performed bulk and single cell RNA-seq of WT and Trem $2^{k 0}$ mice. We show that compared to E4, cortical infusion of E3 lipoproteins upregulated a higher proportion of genes associated with an activated immune response accompanied by a downregulation of homeostatic genes. ScRNA-seq identified microglia-specific clusters affected by Trem2 deficiency suggesting that lack of Trem2 impairs the transition of microglia from homeostatic to an activated state. Compared to E3, E4-expressing microglia showed a reduced $A \beta$ uptake that was additionally aggravated by Trem 2 deficiency. Together, our findings have elucidated unique phenotypic and transcriptional differences in the microglial response to $A \beta$ in the presence of $E 3$ or $E 4$ lipoproteins which could impact $A D$ pathogenesis.

\section{Introduction}

Late-onset Alzheimer's disease (AD) is age-related dementia characterized by amyloid $\beta(A \beta)$ plaques, neurofibrillary tangles and cognitive decline ${ }^{1}$. Inheritance of Apolipoprotein (APOE) $\varepsilon 4$ allele is the major genetic risk factor for late-onset $A D$, modifying disease risk and progression in an isoform dependent manner $\left(\varepsilon 2<\varepsilon 3<\varepsilon 4\right.$, with $\varepsilon 4$ the highest risk) ${ }^{2,3}$. Isoform differences in amino acids at position 112 affects lipid binding properties of APOE and at 158 the receptor-binding affinity to APOE receptors ${ }^{4}$. APOE is a major lipid transporter and the structural differences result in different lipid binding properties. Thus, APOE4 preferentially binds lower density lipoproteins (LDL, VLDL) whereas APOE3 and APOE2 high density lipoproteins $(\mathrm{HDL})^{5}$. In a recent study we applied Shotgun Lipidomics to measure the major phospholipid classes and their molecular spices in brains of AD patients comprising of different APOE genotypes ${ }^{6}$. Our data demonstrated that few of these phospholipids were significantly lower in $A P O E \varepsilon 4 / C$ carriers vs $A P O E \varepsilon 2 / c$ carriers $^{6}$. In the brain, $A P O E$ is primarily secreted by astrocytes and to a lesser extent microglia in a lipid-poor form ${ }^{7}$. ABCA1 transporter mediates the transfer of phospholipids and cholesterol to lipid-free APOE thus forming discoidal high-density lipoprotein (HDL) particles (reviewed $\mathrm{in}^{8}$ ). APOE3 and APOE4 lipoproteins isolated from astrocytes conditioned media (referred to as E3 and E4 lipoproteins) are composed of proteins, cholesterol and phospholipids; and deficient of cholesteryl esters $^{9}$. It has been reported that APOE lipidation state significantly impacts $A \beta$ aggregation ${ }^{10}$ and $A \beta$ clearance at the blood-brain barrier (BBB) ${ }^{11}$. APOE4 isoform is associated with impaired $A \beta$ uptake, 
clearance and degradation ${ }^{12}$ resulting in higher amyloid load in APP transgenic mice ${ }^{13,14}$. Interestingly, a study by Verghese et al. suggested that APOE influences $A \beta$ clearance in vivo not through direct binding but via its interaction with other cell surface receptors ${ }^{15}$.

Microglia can have beneficial effect assisting $A \beta$ clearance and by forming a barrier surrounding amyloid plaques but also they could be detrimental, causing chronic neuroinflammation (reviewed in ${ }^{16,17}$ ). Recent comprehensive single-cell RNA sequencing (scRNA-seq) analysis in mouse models of neurodegeneration discovered a subset of microglia named disease-associated microglia (DAM) ${ }^{18,19}$. The upregulation of DAM genes is usually accompanied by a decreased expression of homeostatic microglia genes responsible to maintain the normal microglia function of surveillance. Among DAM genes are gene variants associated with increased risk for $A D$, such as APOE and TREM2. It has been reported that TREM2 can recognize a variety of ligands including APOE, HDL and LDL which could affect microglial interaction with $A \beta$ and $A P O E^{20,21,22}$. APOE and TREM2 are essential for the development of microglia barrier around the plaques ${ }^{23}$. Recently, we demonstrated that the lack of TREM2 differentially affects the phenotype and transcriptome of human APOE3 and APOE4 expressing mice ${ }^{24}$. TREM2 deficiency increased plaque growth concurrently to the impairment of microglia barrier, an effect most pronounced at earlier stages of amyloid deposition. Interestingly, lack of Trem2 significantly decreased plaqueassociated APOE protein only in APOE4 but not in APOE3 mice in agreement with gene expression data.

In this study, after performing Lipidomic analysis of native E3- and E4-lipoproteins and brain samples from $A P O E \varepsilon 3 / 3$ and $A P O E \varepsilon 4 / 4$ AD patients, we identified a common phospholipid signature between their major phospholipid classes. We hypothesized that isoform-specific phospholipid composition of E3 and E4 native lipoproteins would elicit distinct phenotypic and transcriptomic response by microglia. We used in vivo two-photon imaging and determined that the infusion of $A \beta$ with $E 3$ lipoproteins induced a more rapid microglial response than with E4 lipoproteins. Behavioral testing following cortical $A \beta$ infusion demonstrated that both native lipoproteins ameliorated $A \beta$ deleterious effects on cognition; with APOE3 exhibiting a higher efficacy than APOE4. To determine how E3 and E4 lipoproteins affect microglia expression in response to $A \beta$ we performed bulk and single cell RNA-seq (scRNA-seq). Collectively, the data showed that the addition of $E 3$ lipoproteins to $A \beta$ upregulated a higher proportion of DAM genes and is associated with more active transcriptomic response than E4. scRNA-seq identified microglia-specific clusters affected by Trem2 deficiency suggesting that lack of Trem2 impairs the transition of microglia from homeostatic to activating state. Compared to E3, E4-expressing microglia showed a reduced $A \beta$ uptake that was additionally aggravated by Trem2 deficiency. Together, our findings have elucidated unique phenotypic and transcriptional differences in the microglial response to $A \beta$ when in the presence of either E3 or E4 lipoproteins.

\section{Results}

\section{Common phospholipid signature between APOE\&4/4 and APOEE3/3 AD brains and APOE3 and APOE4 native}




\section{lipoproteins}

Previously we have shown that there is a significant APOE isoform-specific difference in phospholipid content in the brains of AD patients ${ }^{6}$. In the present study, we compared the phospholipid profile of native APOE lipoproteins isolated from conditioned media of APOE3 and APOE4 primary astrocytes to those of $A P O E \varepsilon 4 / 4$ and $A P O E \varepsilon 3 / 3$ brains samples from $A D$ patients. To measure the most abundant nine phospholipid classes in human brain and the native APOE lipoproteins we used Multi-Dimensional Mass Spectrometry Shotgun Lipidomics (MDMS-SL) 6 . We found that out of nine, five phospholipid classes (PE, $\mathrm{PI}, \mathrm{PS}, \mathrm{SM}$ and PA) had a statistically higher level in APOEE3/3 vs APOE\&4/4 brains (Fig. 1a). Overall, with the exception of PG, APOE-containing lipoproteins had a similar direction of fold change for each of class as the human samples (Fig. 1b). In the lipoprotein data set, we found that PI, PS, SM and LPC were statistically higher in E3 vs E4 lipoproteins (for the abbreviation see the legend of Fig. 1). Lipoproteins derived from E3/Abca $1^{\text {het }}$ and $\mathrm{E} 4 / \mathrm{Abca} 1^{\text {het }}$ astrocytes ${ }^{13}$ were used as negative controls and as expected demonstrated half of the phospholipid content of their wild-type counterparts (Suppl. Fig. 1). As shown on Fig. 1c-d, 103 lipid species were commonly identified in the human and lipoprotein samples, most of which were affected in APOE isoform-specific manner in both datasets and were higher in E3 samples. Correlation analysis (Fig. 1e) demonstrated a very strong correlation between the phospholipid profile of human and native lipoprotein samples.

To confirm the results from Shotgun Lipidomics of APOE native particles, we applied liquid chromatography-mass spectrometry (LC-MS) and measured five of the phospholipid classes reported to comprise the bulk of brain APOE-containing lipoproteins ${ }^{9}$ namely: PC, PE, PI, and PS; while CL was used as a control. The tSNE clustering shown on Fig. $1 \mathrm{f}$ demonstrated that the phospholipid molecular species from the two APOE isoforms segregated in separate groups forming distinct clusters. Similar to the MDMS-SL results, we found that the negatively charged phospholipids (PI, PE and PS) were significantly higher in E3 than in E4 lipoproteins but CL was unchanged (Fig. 1g-h). Additionally, specific subspecies of PE and PS (PE36:1, PE36:2, PS36:1, and PS40:6) represented a large portion of the total amount of those classes and were found in significantly less abundance in APOE4 lipoproteins. These specific subspecies may represent potential lipid activation signals which impact receptor binding and could activate signal transduction events.

Since the diameter of lipoproteins is commonly used to represent their lipidation ${ }^{25}$, we used nondenaturing native gel electrophoresis (Fig. 1i) and dynamic light scattering assay (Fig. 1j) to determine the diameter of APOE native particles. The results demonstrated that the size of E3 native lipoproteins peaked at $13 \mathrm{~nm}$ while the size of E4 particles at $12.5 \mathrm{~nm}$ (Fig. 1j). The native gels (Fig. 1i), localized APOE within three populations of particles, migrating around 12, 9 and $8 \mathrm{~nm}$. In agreement with a previous study on $\mathrm{HDL}$, the measurements allowed us to conclude that particles with a diameter between 12 and $9 \mathrm{~nm}$ corresponded to discoidal a-HDL and are lipidated, while particles with a size of $8 \mathrm{~nm}$ corresponded to pre- $\beta$-HDL and are lipid poor/non-lipidated ${ }^{26}$. SDS NuPAGE (Fig. 1i, bottom panel) 
confirmed that an equal amount of APOE protein was used for native gels. Lastly, Suppl. Fig. 1k shows that the cholesterol content of E3 lipoproteins was slightly but significantly increased compared to E4.

\section{APOE lipoproteins rescue $A \beta$-induced memory impairments}

Prior studies demonstrated that the injection of oligomeric $A \beta$ into brain caused significant memory deficits $^{27,28}$. First we tested the effect of native $E 3$ and $E 4$ lipoproteins on $A \beta$ aggregation as before ${ }^{29}$. As visible from Supp. Fig. 2a-c, A $\beta$ incubation with either E3 or E4 native lipoproteins greatly reduced the formation of oligomers. Next, to examine if the addition of native APOE lipoproteins to $A \beta$ ameliorate $A \beta$ induced memory deficits, $A \beta+/-$ APOE (referred to as AbE3 or AbE4) were infused into the cortex of young WT mice followed by behavioral testing as shown on Fig. 2a-d. For both tests, Ab oligomers significantly worsened cognitive performance compared to their corresponding negative control (Fig. $2 b$ and d). In contrast, injections of both AbE3 or AbE4 significantly ameliorated the effects of Ab on cognitive performance. Importantly, the cognitive performance in mice infused with $A \beta E 4$ remained significantly worse compared to their A $\mathrm{B} 3$ counterparts when tested by novel object recognition. For both test, behavior controls showed no significant differences between the groups (Suppl. Fig. 2d-f). The conclusion is that both $E 3$ and $E 4$ native lipoproteins decrease $A \beta$ aggregation and ameliorate its deleterious effects on cognitive performance, with E3 demonstrating a better efficacy.

To investigate the effect of $A \beta E 3$ and $A \beta E 4$ infusions on transcriptome, we injected them in the cortex and performed RNA-seq using sorted microglia and neurons. There were no differentially expressed genes (DEG) when comparing ABE3 vs AßE4 neuronal transcriptomes (Fig. 2e). In contrast, there were 1792 differentially expressed genes (DEG) in microglia: 722 upregulated in mice injected with AßE3 and 1072 genes upregulated in AßE4 injected mice (Fig. 2f, Suppl. Table 2). As shown on Fig. 2g-h, upregulated in A $\beta E 3$ vs A $A$ E4 microglia were many microglia-specific genes (Tmem119 and Trem2) as well as genes and biological processes associated with microglia activation such as cytokines $(C \mathrm{Cl} / 4)$ and catepsins $(C t s d)$.

We also examined the morphology of microglia surrounding the injection site using IBA1 staining and Imaris filament module to trace microglial projections (Fig. 2i-j). The heatmap of microglial projections around the infusion site demonstrated that microglia in the ABE3 group have significantly increased branch length and branch points (bar graph insets) and the projections were oriented more toward the infusion site (the area between heat maps) when compared to the AßE4 group. We also performed macrophage specific F4/80 immunohistochemistry analysis. Fig. 2k-m shows an increased F4/80/Adgre 1 immunostaining around the infusion site in mice injected with ABE3 compared to ABE4, which is consistent with the overall reduction in Adgre 1 gene expression seen in ABE4 microglia. Thus, the results from gene expression and microglia morphology suggest that $A \beta E 3$ induce stronger microglial response when compared to $A \beta E 4$.

\section{Microglia interaction with $A \beta$ is affected by APOE isoform}


To determine if the presence of native E3 and E4 lipoproteins affects microglia function in vivo, we used $\mathrm{Cx} 3 \mathrm{Cr}^{\mathrm{GFP}}$ mice that express EGFP protein in microglia and peripheral monuclear cells and fluorescentlylabeled $A b$. First we tested the lipoproteins impact on $A \beta$ uptake of microglia by injecting AbE3 or AbE4 into the cortex and performing flow cytometry at 4 and $24 \mathrm{~h}$ post-injection (Fig. 3a-c, Suppl. Fig. 3a-d). The cell suspension was separated on microglia without Ab (green signal, single+) and microglia that has engulfed $A b$ (green + red signal, dual+). As shown on Fig. 3b-c, E3 lipoproteins were more efficient than E4 in facilitating $A b$ uptake by microglia at $24 \mathrm{~h}$, but not at $4 \mathrm{~h}$ after the injection.

To further examine if APOE native lipoproteins have an impact on the microglial response to infusion of $\mathrm{A} \beta$, we employed in vivo two-photon imaging in $\mathrm{C} \times 3 \mathrm{cr}^{\mathrm{GFP}}$ mice. For each animal, ZT-stack images were analyzed to quantify the microglial migration towards the infusion site and microglial coverage of the infusion site over time (Fig. 3d-f and Suppl. Fig. 3e-f). The representative images (Fig. 3d and Suppl. Fig. $3 e-f)$ of the time series are color-coded with the initial time point $(0 \mathrm{~min})$ in red and later time points (10 or $80 \mathrm{~min}$ ) in green, and the infused $A \beta$ in white. The time series (Fig. 3d, insets) show clear separation of the green and red labels in the $A \beta E 3$ infusion group, indicative of longer migration and better coverage of the infused $A \beta$ compared to $A \beta E 4$. In contrast, the time series from the mice infused with $A \beta E 4$ demonstrate a visible overlapping - yellow-colored, representing a slower migration velocity and less microglial $A \beta$ coverage. The percent coverage by the microglial processes over time was computed as the increase of the green channel intensity over the infusion site and shown on Fig. 3e. The change in the distance from the microglia to the infusion site is presented on Fig. 3f. Microglia were able to extend processes towards the $A \beta E 3$ infusion over the first 40 minutes. Once the processes reached the $A \beta E 3$, microglia were able to form lamellipodia that covered the ABE3 throughout the remainder of the experiment (40-80 min). In contrast, microglia demonstrated a decreased capacity to extend processes towards the $A \beta E 4$ as well as encapsulate $A \beta E 4$ infusion. The results demonstrate that the infusion of $A \beta$ together with $E 3$ lipoproteins, unlike with $E 4$, induces a more rapid response by microglia to isolate $A \beta$ that suggests a protective mechanism diminishing the spread of $A \beta$.

\section{E3 and E4 lipoproteins differentially affect microglia transcriptome in response to $A \beta$ infusion}

Next, we performed RNA-seq on two FACS sorted microglial populations (dual+ and single+ microglia) from $\mathrm{C} \times 3 \mathrm{cr}{ }^{\text {GFP }}$ mice injected with AbE3 or AbE4 (see Fig. 4a for the design). We assume that dual+ cells are in an active stage compared to their single+ counterparts which are either resting or in transition from homeostatic to active state. The goal was to examine how APOE isoform and the post-injection interval affects this transition. tSNE plots demonstrate that the expression profiles of single+ and dual+ microglia cluster into sharply defined groups for both time points (Fig. 4b). Suppl. Fig. 4a-b demonstrates that numerous overlapping DEGs were commonly upregulated for the two time points in dual+ vs single+ microglia. For each single+ and dual+ populations, we identified genes with a similar level of expression at both time points (unchanged, grey), a group with higher expression at $4 \mathrm{~h}$ that declined in time 
(upregulated at 4h, blue), and a third group (red) with higher expression at 24h (Suppl. Fig. 4c-f). Unchanged in dual+ cells were genes activated from the start with continuous expression and related to interferon signaling (Ifi204) or lipid transport ( $L p /)$. Early responding genes upregulated in dual+ at $4 \mathrm{vs}$ $24 \mathrm{~h}$ were associated with inflammatory response, chemokine-mediated signaling and chemotaxis (Suppl. Fig. 4c-d, blue). Genes that were upregulated at the later time point in dual+ microglia (late responding) were associated with protein folding, ribosome biogenesis and glycolysis consistent with the increased energy demands of processing the engulfed Ab (Suppl. Fig. 4c-d, red color).

As seen from the tSNE plot on Fig. 4b, APOE isoform-specific effect on microglial transcriptomes was more pronounced at $4 \mathrm{~h}$. The examination of DEGs in dual+ vs single+ microglia in both treatments revealed a higher number of total DEGs and specifically a higher number of upregulated genes in dual+ AbE3 microglia than in dual+ AbE4 microglia (Fig. 4c-d, 2760, and 1532 respectively, pie charts dark purple and dark green). For both treatments, there were many shared/common DEGs upregulated in dual+ vs single+ (Fig. 4e-f, grey boxes). In both ABE3 and ABE4 dual+ microglia, there was an overall upregulation of DAM genes (186 genes) and downregulation of the homeostatic genes (212 genes). Commonly upregulated in dual+ microglia were DAM genes (Apoe, $A x l$ ) and downregulated classical homeostatic genes ( $P 2 r y 12$, and Tmem119) indicating a profile characteristic for microglia in an active state (Fig. 4g-h). Commonly upregulated in dual+ were also receptors (Clec4 cluster, Marco, Ax/ and Gas6), cytokines (9 genes) and chemokines (12 genes) and genes related to phagocytosis and lysosomes (Cd68). Surprisingly, Trem2 as well as few other DAM genes (Csf3r, Cebpa and Ctss) had a higher expression in single+ microglia (respectively downregulated in dual+) in both treatments and at both time points. This suggest that Trem2 is more associated with transition than fully activated state. Uniquely upregulated DAM genes for ABE3 were genes associated with lysosome/autophagy process (Lamp1 and Bloc1s1), glycosylation (St6ga/1), which was a glycosyltransferase implicated by GWAS with $\mathrm{AD}^{30}$, cytokines ( $\left.\mathrm{Cl} / 6\right)$ and receptors (Clec4e and $\left.\mathrm{Clec} 12 \mathrm{a}\right)$. DAM genes uniquely upregulated in ABE4 were Capg (binds PIP2 and affects actin dynamic in macrophages ${ }^{31}$ ), Eef2 and Eef1b2 (regulate translation ${ }^{19}$ ) as well as $L y 9$ (lymphocyte antigen $9^{32}$ ). Overall, there was a higher number of DAM genes upregulated in dual+ AbE3 (310) than in dual+ AbE4 (198) (Fig. 4g-h) suggesting that E3 could initiate a stronger response by microglia than $\mathrm{E} 4$.

\section{Single cell sequencing informs on distinct microglial populations in response to $A \beta$ injection}

To test how Trem2 deficiency affects acute microglial response to $A \beta E 3$ or $A \beta E 4$ infused into the cortex of WT and Trem $2^{\mathrm{ko}}$ mice we used scRNA-seq (see Fig. 5a for the design). We collected a total of 49,817 sequenced cells with 2,228 median genes expressed and 5,234 median unique molecular identifiers (UMIs) per cell. Non-microglial cells were identified using cell type-specific genes and excluded from the analysis (Fig. 5b-c, Suppl. Fig. 5) and microglial cells were re-clustered to catalog transcriptionally distinct subpopulations. Thus, we re-clustered 36,244 microglial cells into five clusters and performed trajectory 
analysis to identify distinct activation states in response to the infusion (Fig. $5 \mathrm{~d}$ and Suppl. Fig. 6). We determined three potential trajectories (clusters 2, 3, and 4) from clusters $1 \mathrm{a}$ and $1 \mathrm{~b}$ (Fig. 5d).

To characterize heterogeneity within microglia, we identified cluster-specific genes that are differentially expressed between clusters (Fig. 5e). As seen in Fig. 5e, the microglia in cluster $1 \mathrm{a}$ and $1 \mathrm{~b}$ had increased expression of homeostatic signature genes such as P2ry12, Tmem119, and Siglech ${ }^{19}{ }^{33}$. However, cluster $1 \mathrm{~b}$ showed an increase from the baseline (cluster $1 \mathrm{a}$ ) of the expression of genes related to microglial activation such as Apoe, Cst7, Trem2, and Tyrobp. Thus, we characterized cluster $1 \mathrm{~b}$ as a transitional state. Interestingly, in WT mice Trem2 had the highest expression in cluster 1b suggesting its involvement in the transition from homeostatic to active state. Genes associated with activated microglial were upregulated in clusters 2,3 , and 4 and accompanied by downregulation of the homeostatic microglial genes. This suggests that clusters 2,3, and 4 form in response to infusion of $A \beta E 3$ or $A \beta E 4$ and present the active microglia state. Microglia in cluster 2 showed upregulation of genes related to chemotaxis (Cc/12, Ccl3, Ccl4, Cxcl16, Spp1, and Lgals3), inflammatory cytokine (Mif), lipoprotein lipase (LpI) and ribosomal components. Microglia in cluster 3 showed upregulation of pro-inflammatory genes, especially a set of genes important in NF- B signaling such as Tnf, $/ 1$, and Ptgs2, as well as immediate early genes (Fos, Jun, and Egr1) and cytokines ( $C \mathrm{Cl} / 3$ and $C \mathrm{Cl} / 4$ ). In cluster 4, genes related to the interferon signaling pathway including Ifitm3, Ifi27/2a, Ifit1, Irf7 as well as Apoe were highly expressed with top enriched GO term "immune system process" (Fig. 5e, Suppl. Table 3). In both genotypes, the highest proportion of cells was found in cluster $1 \mathrm{~b}$ (transitional state) and cluster 2 (active state). We observed an increased proportion of cells in cluster $1 \mathrm{~b}$ and reduced in cluster 2 in Trem2-deficient microglia compared to WT (Fig. 5f). This suggests that Trem2-deficiency impairs the transition of microglia from homeostatic to activating state particularly from cluster $1 \mathrm{~b}$ to cluster 2 .

\section{scRNA-seq identifies activated gene clusters downregulated in Trem $2^{\mathrm{ko}}$ mice following $A \beta$ infusion}

To identify differential transcripts, we compared microglia from Trem $2^{\mathrm{ko}}$ and WT mice and observed a higher number of DEGs in clusters 2, 3, and 4 associated with active microglia (Fig. 6a, Suppl. Table 4). Overall, there was reduced expression of immune response genes in Trem2 deficient microglia compared to WT microglia (Fig. 6b-d). In clusters 2, 3, and 4, DAM genes comprised $30-44 \%$ of the total number of differentially expressed genes that were upregulated in WT vs Trem $2^{\mathrm{ko}}$ and this was accompanied by the same decrease of homeostatic microglia genes (Fig. 6b-d) suggesting that microglia from Trem $2^{\mathrm{ko}}$ mice cannot adequately respond to the $A \beta E 3 / A \beta E 4$ infusion challenge. In Cluster 2 top downregulated genes in Trem $2^{\mathrm{ko}}$ microglia were $L p l, S p p 1, C d 9$ and opposed by upregulation of canonical homeostatic genes such as P2ry 12 (Fig. 6b). Clusters 3 (Fig. 6c) and Cluster 4 (Fig. 6d) exhibited the largest number of down regulated genes in Trem $2^{\mathrm{ko}}$ microglia and also showed the highest mismatch between WT and Trem $2^{\mathrm{ko}}$ microglia in terms of homeostatic/DAM gene expression (see the bar graphs in Fig. $6 \mathrm{c}$ and d). 
We validated the scRNA-seq data using fluorescent in situ hybridization (FISH) for Tmem119 and Adgre1 and spatially visualized their expression near the infusion site. We selected Tmem119 as a canonical microglia-specific gene that was not affected by Trem2 deficiency and Adgre1 (F4/80) was selected because it was significantly downregulated in Trem $2^{\mathrm{ko}}$ microglia and we showed to be increased near the infusion site (see Fig. 2k-i). Similarly, to the scRNA-seq data we found a significant reduction in gene expression of Adgre 1 in Trem $2^{\mathrm{ko}}$ mice and no effect on Tmem 119 level (Fig. 6e-g). Thus, FISH performed near the infusion site confirms the result for selected genes in the scRNA-seq data sets.

\section{Trem2 deficiency and APOE isoform affects microglia response to $A \beta$}

We next examined if the response to $A \beta E 3$ and $A \beta E 4$ infusion is APOE isoform- and/or Trem2-dependent. We compared microglia from $A \beta E 3$ and $A \beta E 4$ injected WT and Trem $2^{\mathrm{ko}}$ mice separately within clusters 2 , 3 and 4 which correspond to active microglia (Fig. 7a-C). We determined that in WT mice, clusters 2 and 3 contained a higher number of DEGs as well as DAM genes in ABE3 treated mice than in ABE4 and the opposite was detected for cluster 4 . For all clusters, while $A \beta E 3$ injection upregulated a comparable number DEGs in Trem2-deficient microglia as in WT, AßE4 injection resulted in significantly less DEGs in Trem $2^{\mathrm{ko}}$ microglia (Fig. 7a-c). In cluster 2, Trem2 ${ }^{\mathrm{ko}}$ mice injected with $\mathrm{A} \beta E 4$ had a higher expression of classic homeostatic genes such as P2ry 12 and P2ry 13 vs their E3 counterparts (Fig. 7a, Trem2 ${ }^{\text {ko }}$ plot) and this was not observed in WT microglia (Fig. 7a, WT plot). On the other hand, there were many overlapping DEGs commonly upregulated in WT and Trem $2^{\mathrm{ko}}$ mice injected with AbE3 such as Spp1 (Fig. 7a). In cluster 4 , there were more homeostatic genes ( $C \times 3 c r 1, C s f 1 r)$ upregulated in WT- AßE3 mice than in WT-AbE4 mice (Fig. 7c) and the opposite was observed for DAM genes that were upregulated in WT-AbE4 mice (Ifi27/2a, Eef1g). However, for Cluster 4 we identified very few DEGs in Trem2 ${ }^{\text {ko }}$ mice. Overall, these results suggest that Trem2 deficiency may further exaggerate E4 lipoprotein insufficiency to activate microglia.

To test how Trem2 deficiency and APOE native particles affect $A \beta$ uptake we used primary microglia from mice expressing human E3 and E4; and compared to their Trem $2^{\mathrm{ko}}$ counterparts (E3/Trem2 ${ }^{\mathrm{ko}}$, or E4/Trem2 ${ }^{\mathrm{ko}}$ ). As seen on Fig. 7d-f, both APOE4 isoform and Trem2 status significantly decreased A $\beta$ uptake. As visible, the overall reduction of $A \beta$ uptake by $E 4 / T r e m 2^{k o}$ microglia was reduced 5 -fold compared to E3 expressing microglia. Interestingly, Trem2 deficiency significantly affected the morphology of the microglia as its shape become more circular with less processes (Fig. 7f). These results confirm that $\mathrm{E} 3$ is more successful than $\mathrm{E} 4$ at promoting $\mathrm{A} \beta$ uptake and that Trem 2 deletion further aggravates E4 mediated inefficiency.

\section{Discussion}


In this study, we identified a common phospholipid signature between major phospholipid classes of native $\mathrm{E} 3$ and $\mathrm{E} 4$ lipoproteins and brain samples from $A P O E \varepsilon 3 / 3$ and $A P O E \varepsilon 4 / 4$ AD patients. Our results support the hypothesis that native E3 lipoproteins trigger a more effective microglial response to $A \beta$ than $E 4$ in ameliorating $A \beta$ deleterious effect. We posit that the divergence in phospholipid content of $E 3$ and $E 4$ native lipoproteins affects $A \beta$ binding to microglia receptors in isoform-specific manner and consequently have a differential impact on microglia transcriptome (Fig. 8). Consequently, E3 induces a faster microglial response than E4 characterized by rapid process extension allowing $A \beta$ to be isolated and engulfed. Lastly, behavioral testing following $A \beta$ injections demonstrated that $E 3$ native lipoproteins exhibited a better protection than $E 4$ in diminishing $A \beta$ effects on memory.

The quantitative assessment of the phospholipid composition of E3 and E4 native lipoproteins and brain samples from $A P O E \varepsilon 3 / 3$ and $A P O E \varepsilon 4 / 4$ AD patients demonstrated a significant APOE isoform-specific difference in phospholipid profiles. Interestingly, our analysis demonstrates a very strong correlation of the phospholipid profile of human and native lipoprotein samples (Fig. 1e). It should be noted that the human brain samples include lipids that are structural part of cellular membranes as well as lipoproteins in interstitial fluid. Regardless that the source of the phospholipids was not entirely the same, it was remarkable to discover such high level of correlation between the abundance and the direction of fold change between the phospholipids of human samples and the native lipoproteins isolated from astrocyte conditioned media. As the major lipid carrier in the brain APOE lipoproteins play an important role in the chaperoning of cholesterol and phospholipids between cells and serving as ligand for multiple immune receptors such as TREM2. It has been shown that TREM2 signaling is activated by phospholipids such as phosphatidylinositol and phosphatidylcholine ${ }^{34}$ and TREM2 binds APOE as well as LDL and HDL ${ }^{22}$. Our lipidomic results demonstrate that four major phospholipid classes including neutral (PC and $\mathrm{PI}$ ) and negatively charged (PE and PS) are significantly reduced in E4 lipoproteins when compared to E3 but not cardiolipin. Sensing lipids is important for microglia activation and affects functions such as chemotaxis and phagocytosis (reviewed in ${ }^{35}$ ). Increasing evidence suggests that phospholipid levels decrease with aging as well we during $A D$ progression ${ }^{36}$, making the initial deficit we have identified in $E 4$ particles even more detrimental with the progression of the pathology. The reduced lipidation of E4 compared to E3 native particles may impact $A \beta$ aggregation which in turn could affect cognitive performance and microglia activation state ${ }^{37}$. Numerous studies demonstrated APOE binds to Ab with high affinity and most agree that there is an isoform-specific effect on Ab aggregation E4>E3>E2 ${ }^{38}$, 39. Furthermore, studies have suggested that $\mathrm{E} 3$ forms more stable complex with $\mathrm{Ab}$ than $\mathrm{E} 4^{39}$ however in the present study we did not examine the stability of Ab-APOE complex. Our data demonstrated that both E3 and E4 native lipoproteins precluded the formation of Ab oligomers (Suppl. Fig. 2a-b). However, we cannot exclude a possible presence of occasional Ab oligomers and potentially an isoform-specific effect on $A b$ oligomerization that could be detected using quantitative methods with higher resolution. In confirmation of such possibility is the fact that E3 was more effective than E4 in rescuing cognitive deficits caused by Ab oligomers (Fig. 2a-d). 
In terms of APOE effect on microglia we found that E3 native lipoproteins are more effective than E4 in triggering microglia response to $A b$ injections. The transcriptomic profile of microglia isolated from $A \beta E 3$ injected mice have a higher enrichment of gene expression networks associated with innate immune response compared to microglia from $A \beta E 4$ injected. To address the transcriptional changes in phagocytic and non-phagocytic microglia we compared dual+ vs single+ at 4- and 24-hours postinjection. Transcriptionally, the dual+ populations are strikingly different from single+ at both 4- and 24hours post-injection, representing an activated state with upregulation of DAM genes and downregulation of homeostatic genes. We further sub-divided dual+ population into early responders (4h; Cc/9, Ifit 1 , and $C t s b$ ), genes with continuous expression (Ifi204, Lpl, and //10), or late responders (24h; Adgre1, Mif, and Cc/2). By separating of microglia on dual+ and single+ we conclude that that the APOE- isoform-specific effects are more pronounced at earlier points of the injury that may reflect involvement in the initial recognition of Ab+APOE complexes by microglia. Furthermore, in Cx3cr1 mice (Fig. 4), A EE3 injections were associated with more active transcriptomic profile comprised by differential increase of DAM compared to $A \beta E 4$ injections.

These transcriptional changes are accompanied by phenotypic changes in microglial morphology exhibited by increased process orientation towards the infusion site in $A \beta E 3$ vs $A \beta E 4$ injected mice and an increase in microglia which colocalized with $A \beta$. We confirmed this using in vivo two-photon imaging, demonstrating that within the first hour of infusion microglia exposed to $A \beta E 3$ extended processes toward $A \beta$ faster than $A \beta E 4$. Thus, in brains injected with $A \beta E 3$, microglia can cover and envelop $A \beta$ with better efficiency that microglia from $A \beta E 4$ injected brains (Fig. 3). Finally, in vivo and in vitro experiments show that E3-native particles are more effective than $E 4$ at promoting $A \beta$ uptake (Fig. 3 and 7). Our interpretation of these findings is that $\mathrm{E} 3$ is better at mobilizing microglia to form a protective barrier surrounding the neuronal damage caused by the $A \beta$ injection and restrict its spread by increasing $A \beta$ uptake.

Recently we reported that microglia-plaque coverage of small plaques is more efficient at restricting plaque-growth than of larger plaques ${ }^{24}$, and this effect is modulated by both APOE isoform as well as TREM2 status. This emphasizes the importance of the early response by microglia to amyloid and implicates the phenotypic response seen in the first 24 hours in the AßE3 mice as critical to the long-term protection from amyloid buildup and eventual neuronal damage. Studies addressing the microglia phenotypic response in TREM2 deficient mice have shown impaired ligand binding, reduced cell clustering to amyloid, reduced plaque compaction, and an impaired activation profile ${ }^{19,21,24,34,40}$. Using scRNA-seq we identify clusters 2, 3 and 4 as infusion-responsive clusters characterized by upregulation of DAM genes and downregulation of homeostatic microglial genes, a profile that has been reported in neurodegenerative diseases ${ }^{19,41}$. In these clusters, Trem $2^{\mathrm{ko}}$ microglia when compared to WT microglia show a significant downregulation of DAM genes and upregulation of homeostatic genes (Fig. 6). Finally, within the activated clusters we find less difference between APOE isoforms in Trem2 ${ }^{\mathrm{ko}}$ mice possibly resulting from the impaired ability of Trem $2^{\mathrm{ko}}$ microglia to recognize ligands. 
Collectively our findings have identified that in the presence of $A \beta$, E3 lipoproteins are more effective at providing rapid transcriptional and phenotypic response than E4 lipoproteins that can counteract $A \beta$ harmful effects.

\section{Methods}

\section{RESOURCE AVAILABILITY}

\section{Lead Contact}

Further information and requests for resources and reagents should be directed to and will be addressed by the Lead Contact, Rada Koldamova (radak@pitt.edu).

\section{Materials Availability}

This study did not generate new unique reagents.

\section{Data and Code Availability}

The RNA-seq expression data has been deposited in the GEO database under the accession number: To Be Determined.

\section{METHOD DETAILS}

\section{Experimental models}

All animal experiments were performed in accordance with the NIH Guide for Care and Use of Animals and approved by the University of Pittsburgh Institutional Animal Care and Use Committee. The following mouse lines were bred to generated experimental mice: Wild-type C57BL/6J (WT; JAX), human APOE3

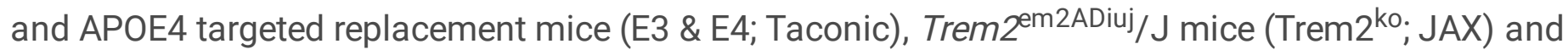
129P2(Cg)-Cx3 $\mathrm{cr}^{\text {tm1 } 1 \mathrm{Litt} / J}$ (Cx3cr1 ${ }^{\text {GFP }}$; JAX). Human APOE3 and APOE4 targeted replacement mice were

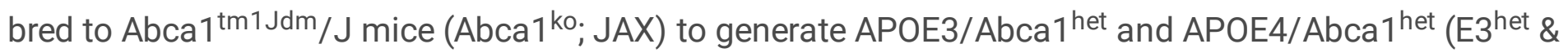
E4 $\left.{ }^{\text {het }}\right)$. Likewise, human APOE3 and APOE4 targeted replacement mice were bred to Trem $2^{\text {em2ADiuj} / J ~ m i c e ~}$ (Trem2 ${ }^{\mathrm{ko}} ; \mathrm{JAX}$ ) to generate APOE3/Trem2 ${ }^{\mathrm{ko}}$ and APOE4/Trem2 ${ }^{\mathrm{ko}}$ (E3/Trem2 ${ }^{\mathrm{ko}}$ \& E4/Trem2 ${ }^{\mathrm{ko}}$ ). All experimental mice were on the C57BL/ 6 genetic background, kept on a $12 \mathrm{~h}$ light-dark cycle with ad libitum access to food and water and randomly assigned to an experimental group. All reagents were purchased from Fisher Scientific unless documented otherwise. 


\section{AD brain samples}

Human samples (Suppl. Table 1) were provided by the University of Pittsburgh Alzheimer's Disease Research Center (ADRC) brain bank and the Sanders-Brown Center on Aging at the University of Kentucky. Braak staging was performed on Bielschowsky-stained sections and $A P O E$ allelic polymorphism determined by a PCR-based assay. Gray matter samples of $A P O E \varepsilon 3 / 3$ and $A P O E \varepsilon 4 / 4$ genotypes from the right inferior parietal lobule were dissected and used for Multi-Dimensional Mass Spectrometry Shotgun Lipidomics (MDMS-SL). Age and Postmortem intervals (PMI) matching was confirmed by t test.

\section{Native APOE generation and characterization}

Cultures of primary astrocytes were established from one-day-old E3, E4, E3 ${ }^{\text {het }}$ and E4 ${ }^{\text {het }}$ targeted replacement pups as previously shown ${ }^{42,43}$. Briefly, the cortices and hippocampi were mechanically dissociated using a sterile pasteur pipette and cultivated in DMEM/F12 medium supplemented with 10\% bovine growth serum, l-glutamine and antibiotics. Cells were cultured on poly-d-lysine $(100 \mu \mathrm{g} / \mathrm{mL})$ coated T75 Costar flasks (Corning). Confluent primary astrocytes were incubated with treatment medium (Neurobasal medium with antibiotics and glutamine as above minus serum) for $48 \mathrm{~h}$. Conditioned medium were collected, immediately filtered through $0.22 \mu \mathrm{m}$ filter and concentrated using Amicon $\circledast$ Ultra Centrifugal Filters (10kD cutoff). Native APOE lipoproteins were washed 3 times with cold PBS and their concentration measured by quantitative Western Blotting (NUPAGE) using commercial human APOE (Meridian Life Science) as a standard. Native APOE particles were resolved on Novex ${ }^{\mathrm{TM}} 4-20 \%$ Tris-Glycine gels using an Amersham ${ }^{\text {TM }}$ HMW calibration kit as native ladder (GE Healthcare). The size of APOE lipoprotein particles were measured by dynamic light scattering with Zetasizer Nano Z (Malvern). APOE lipoprotein cholesterol content was assessed with the Cholesterol Quantitation Kit (Sigma) according to the manufacturer's instructions.

\section{Multi-Dimensional Mass Spectrometry Shotgun Lipidomics (MDMS-SL)}

The MDMS-SL assay 44, 45, 46 was performed to determine differences in the lipid composition of the native E3 and E4 particles isolated from primary astrocyte cultures and human AD brain samples described above. APOE particles isolated from $E 3^{\text {het }}$ and $E 4^{\text {het }}$ primary astrocytes, exhibiting diminished APOE lipidation states, were used as negative controls. Quantitative analysis was performed on a triplequadrupole mass spectrometer (Thermo Fisher Scientific) equipped with an automated nanospray apparatus NanoMate and Xcalibur system. Internal standards for the quantification of individual molecular species of the major lipid classes were added to each sample at the start of the extraction procedure. Lipid extraction was performed by the methyl-tert-butyl ether (MTBE) method with resuspension in chloroform/methanol $(1: 1 \mathrm{v} / \mathrm{v})$ solution with nitrogen flush. Identification and quantification of all reported lipid molecular species were performed using an in-house automated software program ${ }^{45}$. The resulting MDMS-SL data was analyzed and visualized in $\mathrm{R}(\mathrm{v} 3.6 .0)$ and GraphPad Prism (v8.3.1). Phosphatidylcholine = PC; Phosphatidylinositol = Pl; 
Phosphatidylethanolamine = PE; Phosphatidylserine = PS; Sphingomyelin = SM; Lysophosphatidylcholine = LPC; Lysophosphatidylethanolamine = LPE; Phosphatidylglycerol = PG; and Phosphatidic acid = PA.

\section{Liquid Chromatography-Mass Spectrometry (LCMS) Lipidomics}

Phospholipids were extracted and separated as before ${ }^{47}$. Briefly, MS analysis of phospholipids was performed on a Q-Exactive hybrid-quadrupole-orbitrap mass spectrometer (Thermo Fisher Scientific) as previously described ${ }^{47}$ using a normal phase column (Luna $3 \mu \mathrm{m}$ Silica $100 \AA, 150 \times 2.0 \mathrm{~mm}$, (Phenomenex)) at a flow rate of $0.2 \mathrm{~mL} / \mathrm{min}$ on a Dionex Ultimate $3000 \mathrm{HPLC}$ system (maintained at $35^{\circ} \mathrm{C}$ ). The analysis was performed using $A$ and $B$ gradient solvents containing $10 \mathrm{mM}$ ammonium acetate and 0.5\% triethylamine (A - propanol:hexane:water (285:215:5, v/v/v) and B propanol:hexane:water $(285: 215: 40, \mathrm{v} / \mathrm{v} / \mathrm{v}))$. The column was eluted for $0.5 \mathrm{~min}$ isocratically at $25 \% \mathrm{~B}$, then from 0.5 to 6.5 min with a linear gradient from $25 \%$ to $40 \%$ solvent B, from $6.5-25$ min using a linear gradient of $40-55 \%$ solvent $B$, from $25-38$ min with a linear gradient of $55-70 \%$ solvent $B$, from $38-48$ min using a linear gradient of $70 \%-100 \%$ solvent $B$, then isocratically from $48-55 \mathrm{~min}$ at $100 \%$ solvent $B$ followed by a return to initial conditions from $55-70$ min from $100 \%$ to $25 \%$ B. The column was then equilibrated at $25 \% \mathrm{~B}$ for an additional $5 \mathrm{~min}$. Analysis was performed in negative ion mode at a resolution of 140,000 for the full MS scan in a data-dependent mode. The scan range for MS analysis was $400-1800 \mathrm{~m} / \mathrm{z}$ with a maximum injection time of $128 \mathrm{~ms}$ using 1 microscan. An isolation window of 1.0 Da was set for the MS and MS2 scans. Capillary spray voltage was set at $3.5 \mathrm{kV}$ and capillary temperature was $320^{\circ} \mathrm{C}$. The S-lens Rf level was set to 60 . The phospholipid content for each APOE isoform was normalized to the amount of APOE protein as determined by SDS NUPAGE. The analysis of the resulting LCMS data was performed and visualized in R (v3.6.0) and GraphPad Prism (v8.3.1). See lipid class abbreviations in prior section.

\section{$A \beta$ oligomer preparation}

$A \beta_{42}$ peptide (American Peptide Company), HiLyte ${ }^{\mathrm{TM}}$ Fluor $488-A \beta_{42}$ or HiLyte ${ }^{\mathrm{TM}}$ Fluor $555-\mathrm{A} \beta_{42}$ (AnaSpec) were used for all $A \beta$ oligomerizations and injections as before ${ }^{27}$. Under a fume hood, $0.1 \mathrm{mg} A \beta$ peptide was dissolved in ice cold 1,1,1,3,3,3-Hexafluoro-2-Propanol (HFIP, Fluka) then vortex for a few seconds. The solution was dried with a gentle stream of nitrogen to obtain a peptide film at the bottom of the vial. Prior to use, the film was re-suspended in anhydrous dimethyl sulfoxide (DMSO) to form a $5 \mathrm{mM}$ solution, sonicated in a water bath for $10 \mathrm{~min}$ and diluted in sterile phosphate-buffered saline (PBS) to a final concentration of $100 \mu \mathrm{M}$. HiLyte ${ }^{T M}$ Fluor 488 - or $555-A \beta$ or $A \beta_{42}$ peptide and E3 or E4 native particles were combined, vortexed, held under oligomer forming conditions (room temperature) for $24 \mathrm{~h}$ at a final concentration of $50 \mu \mathrm{M} \mathrm{AB}$ and $5 \mu \mathrm{M}$ APOE and stored at $-20^{\circ} \mathrm{C}$ until use (abbreviated ABE3 or ABE4). The same molar concentration (10:1) of scrambled $A \beta$ (AnaSpec) was dissolved in vehicle, combined with isolated E3 or E4 particles, held under oligomer forming conditions and stored at $-20^{\circ} \mathrm{C}$ until use (abbreviated scrA $\beta E 3$ or scrAßE4). scrA $3 E 3$ or scrA $\beta E 4$ was utilized as negative controls throughout the 
subsequent experiments. Furthermore, $A \beta_{42}$ peptide and scrambled $A \beta$ was incubated with PBS vehicle as negative control for APOE particles.

\section{$A \beta$ oligomer characterization}

\section{Western Blot}

$A \beta$ oligomers were examined using western blotting, proteins were resolved on $4-12 \%$ Bis-Tris gels (Invitrogen) and transferred onto nitrocellulose membranes. These membranes were probed with 6E10 antibody (Biolegend) and anti-APOE antibody (Invitrogen) with immunoreactive signals visualized using enhanced chemiluminescence.

\section{Electron Microscopy}

For electron microscopy, $5 \mu \mathrm{L}$ of each sample was placed on a freshly glow-discharged carbon-coated grid, adsorbed for 2 min and excess solution was blotted using filter paper ${ }^{27}$. The grid was washed with deionized water before staining with $5 \mu \mathrm{L}$ of freshly filtered uranyl acetate solution $(1 \%, w / v)$ for $15 \mathrm{~s}$. Excess stain was blotted and the grid was allowed to air-dry. Grids were imaged on a Tecnai T12 microscope (FEI) operating at $120 \mathrm{kV}$ and $\times 30,000$ magnification and equipped with an UltraScan 1000 CCD camera (Gatan) with post column magnification of $1.4 \mathrm{x}$.

\section{Guide cannula implantation}

To examine the cognitive effects in WT male mice ( 3 mo.), A $\beta$ oligomers co-incubated with native APOE lipid particles were infused directly into the brain through implanted guide cannulas ${ }^{27}$. Following anesthesia with isoflurane, the head was shaven and sterilized with two separate povidone-iodine alcohol washes. A 50\% mixture of bupivacaine and lidocaine was applied to the surgical site and ophthalmic ointment applied to the eyes. The head was leveled in a stereotaxic frame and an incision made exposing the dorsal aspect of the skull. Two holes were drilled into the skull (coordinates: AP=-2.46 $\mathrm{mm}, \mathrm{L}= \pm 1.50 \mathrm{~mm}$ ) and 26-gauge guide cannulas (Plastics One) were lowered to a depth of $1.0 \mathrm{~mm}$. Cannulas were fixed to the skull with acrylic dental cement attached to two bone anchoring screws and the surgical opening sutured closed. After suturing, animals were administered buprenorphine and sterile saline, body temperature was maintained until sterna recumbence and animals were allowed to recover for 8 days prior to the start of behavioral testing.

\section{Behavioral testing}

All stages of behavioral testing were performed at the same time of the day (during the light phase), ensuring $24 \mathrm{~h}$ between each phase of testing. Fear conditioning was started $24 \mathrm{~h}$ after the completion of novel object recognition. Prior to behavioral testing, mice were placed into individual containers, taken to 
the behavioral testing room and handled for $3 \mathrm{~min}$ for three consecutive days to reduce anxiety. Thirty minutes before a training stage, animals received an infusion of $A \beta$ oligomer co-incubated with either $E 3$ or E4 (Fig. 2a-d). Scrambled A $\beta$ co-incubated with either E3 or E4 was used as a negative control. Mice were randomly assigned to either the $A \beta E 3, A \beta E 4$, scrA $A E 3$ or scrA $\beta E 4$ group. The dummy cannulas were removed and infusion cannulas, attached to microsyringe pump by polyethylene tubing, were placed in the guide cannula. $A \beta$ oligomer (final volume of $1 \mu \mathrm{L}$ per hemisphere) was infused over $1 \mathrm{~min}$, the cannulas were left in place for $1 \mathrm{~min}$ to allow for diffusion of the sample and finally dummy cannulas replaced. Each animal received 5 infusions of $A \beta$ oligomer starting on day 2 of novel object recognition until the completion of behavioral testing (day 6). Between behavioral trials, the paradigms were cleaned with $70 \%$ ethanol to eliminate any olfactory cues. Performance was recorded and scored using ANY-maze software (Stoelting Co.) during all phases of testing.

\section{Novel Object Recognition}

Novel object recognition (NOR) was performed over three consecutive days as previously described ${ }^{24}$. On Day 1, habituation phase, each animal was allowed to freely explore an open arena $(40 \mathrm{~cm} \times 40 \mathrm{~cm} \times 30$ $\mathrm{cm}$ white plastic box) for two 5 min trials with a 5 min inter-trial interval. On Day 2, familiarization phase, each animal was returned to the arena containing two identical objects (tower of LEGO ${ }^{8}$ bricks) located in opposite diagonal corners for two 5 min trials separated with a 5 min inter-trial interval. On Day 3 , testing phase, the animal was returned to the arena with two objects in the same positions as previously, but one object was replaced with a novel object (metal bolt and nut of similar size). Mice were allowed to explore the two objects for $10 \mathrm{~min}$. The exploration of both objects was defined as the mouse sniffing or interacting while facing an object within $3 \mathrm{~cm}$. Mice were consistently placed into the middle of the arena facing the posterior wall to prevent any object preference. The percent exploration, an indicator of recognition memory, was determined by dividing the time exploring the novel object by the total time exploring both objects. Locomotor activity was assessed by measuring the total distance traveled in the open field during day 1 of testing.

\section{Contextual and Cued Fear Conditioning}

Contextual and Cued Fear Conditioning (CCFC) was performed over three consecutive days as previously described $^{24}$. On Day 1 , training phase, mice were placed in a conditioning chamber (Stoelting Co.) for 5.5 min. The first 2 mins were silent, allowing the mouse to acclimate to the chamber; this was followed by a $30 \mathrm{sec}$ tone $(2,800 \mathrm{~Hz}$; Intensity $85 \mathrm{~dB}$, conditioned stimulus (CS)) ending in a 2 sec foot shock $(0.7 \mathrm{~mA}$, unconditioned stimulus (US)) through the floor of the conditioning chamber. The process was repeated one more time and ended with $30 \mathrm{sec}$ of re-acclimation. On Day 2, contextual phase, mice were placed in the same conditioning chamber for 5 min with no tone or shock administered, to measure contextual fear conditioning. On Day 3, the gray walls of the chamber were replaced with black and white striped walls to introduce a novel environment for assessing cued fear conditioning. Mice were placed in the conditioning chamber for $5 \mathrm{~min}$. After the first $2 \mathrm{~min}$ of silence, the tone was administered for $3 \mathrm{~min}$, to measure cued 
fear conditioning. Freezing time was defined as the absence of movement except for respiration and calculated as percent freezing of the total time in the chamber during each phase of testing.

\section{Cortical infusion}

WT male mice (3 mo.) received cortical infusions of un-labeled $A \beta E 3$ or $A \beta E 4$. In a separate cohort of $\mathrm{C} \times 3 \mathrm{Cr}^{\mathrm{GFP}}$, WT and Trem2 ${ }^{\mathrm{ko}}$ male mice (3 mo.), HiLyte ${ }^{\mathrm{Tm}}$ Fluor 555 -labeled A $\beta$ combined with native $\mathrm{E} 3$ and $E 4$ particles was infused into the cortex. Mice were randomly assigned to either the scrambled $A \beta, A \beta$ alone, $A \beta E 3$ or $A \beta E 4$ group. For the bilateral infusion, a 28-gauge infusion cannula (Plastics One) was connected to a $10 \mu \mathrm{L}$ glass syringe (Hamilton) with vinyl tubing and placed in a micro syringe pump. Following anesthesia with isoflurane, the head was shaven and sterilized with two separate povidoneiodine-alcohol washes. A 50\% mixture of bupivacaine and lidocaine was applied to the surgical site and ophthalmic ointment applied to the eyes. The head was leveled in a stereotaxic frame and an incision made, exposing the dorsal aspect of the skull. Four holes were drilled into the skull (coordinates: $A P=-1$ and $-2.5 \mathrm{~mm}, \mathrm{~L}=+/-2.0 \mathrm{~mm}$ ) and the infusion cannula was lowered into the cortex ( $\mathrm{DV}=-1.0 \mathrm{~mm})$. At each infusion site, $2 \mu \mathrm{L}$ of $A \beta$ preparation was infused at a rate of $0.5 \mu \mathrm{L} / \mathrm{min}$ and the cannula remained in place for 4 min following the infusion. Following the infusions, the surgical opening was sutured closed, animals were administered buprenorphine and sterile saline and placed on a heating pad until fully recovered.

\section{Animal tissue processing}

4 or $24 \mathrm{~h}$ following the cortical infusion, mice were perfused and tissues collected. Mice were anesthetized with Avertin ( $250 \mathrm{mg} / \mathrm{kg}$ of body weight, i.p.) and transcardially perfused with $25 \mathrm{~mL}$ of cold $0.1 \mathrm{M}$ PBS, $\mathrm{pH}$ 7.4. For $\mathrm{C} \times 3 \mathrm{cr} 1^{\mathrm{GFP}}$ and $\mathrm{Trem} 2^{\mathrm{ko}}$ mice used for FACS, flow cytometry and single cell RNAseq the infusion sites in both hemispheres were excised for cellular isolation as described below. For WT mice, one hemisphere was drop fixed in $4 \%$ phosphate-buffered paraformaldehyde at $4^{\circ} \mathrm{C}$ for $48 \mathrm{~h}$ before storage in $30 \%$ sucrose. The other hemisphere was used for cellular isolation as described below. Fixed hemibrains were mounted in O.C.T. and cut in the coronal plane at $30 \mu \mathrm{m}$ sections using a frozen cryotome (Thermo Scientific) and stored in glycol-based cryoprotectant at $-20^{\circ} \mathrm{C}$ until histological staining. Prior to staining, sections containing the infusion site were selected under a dissection microscope (Olympus).

\section{Immunohistochemistry}

To characterize changes in activated microglia in the vicinity of the infusion site, a series of 6 brain sections from each animal was immunostained with anti-IBA1 antibody (WAKO). Free-floating sections were washed, followed by antigen retrieval in sodium citrate buffer at $80^{\circ} \mathrm{C}$ for $60 \mathrm{~min}$, blocked in Normal Donkey Serum (Jackson Lab) for $1 \mathrm{~h}$ and finally incubated in IBA1 antibody $(1: 1000)$ overnight at $4^{\circ} \mathrm{C}$. 
Sections were washed and transferred into secondary donkey anti-rabbit Alexa 594 antibody (Invitrogen) for $1 \mathrm{~h}$, before being washed, mounted on superfrost plus slides and coverslipped. Fluorescent confocal images of the infusion site were taken using a Nikon A1 confocal microscope at 60x magnification with $1.0 \mu \mathrm{m}$ step size. Four sequential images were captured flanking either side of the infusion site. Analysis was performed as in Marsh et al. ${ }^{48}$ with modifications. The FilamentTracer module (Imaris, version 7.1.1, Bitplane) was utilized to trace processes of cortical IBA1 positive microglia and determine process length and number of branch points as indicators of microglial morphology complexity. Using the 3D mapped filament tracings, we oriented all microglia so their central axis was positioned facing the infusion site and generated a heatmap depicting the location of microglia from an experimental group utilizing the ImageJ - heatmap from image stack plugin (National Institutes of Health). The number of overlapping microglial processes increases the pixel saturation metric. The percent of all processes which occupy space in each of the 4 quadrants was used to determine the percent coverage of that quadrant.

A second series of 6 brain sections around the infusion site was used for F4/80 immunostaining. First, we quenched endogenous peroxidases with $0.3 \%$ hydrogen peroxide, tissues were blocked in $3 \%$ normal goat serum (Vector), then blocked for endogenous avidin and biotin. Sections were incubated in F4/80 antibody (Abcam) overnight at $4^{\circ} \mathrm{C}$. Sections were washed and transferred into secondary biotinylated anti-rat antibody (Vector) for 90 min before being washed and subsequently developed using the Vector $A B C$ kit and $D A B$ substrate kit (Vector). Sections were mounted onto superfrost plus slides and coverslipped. Bright-field images were taken using a Nikon Eclipse 90i microscope at 20x magnification encompassing the infusion site. Image intensity threshold was established to detect the F4/80 staining compared to background using NIS Elements software (Nikon Instruments Inc.) and values were represented as the area of staining normalized to total image area or percentage of area covered.

\section{Magnetic-activated cell sorting (MACS)}

For WT mice infused with unlabeled $A \beta$, we isolated microglial and neuronal cellular populations utilizing MACS column-based protocols according to manufacturer instructions (Miltenyi Biotec). First, the tissue was dissociated utilizing the Neural Tissue Dissociation kit (Miltenyi Biotec), a gentle two-step enzymatic dissociation (papain and trypsin) that yields a high number of viable cells. Briefly, the cortical tissue was enzymatically and mechanically lysed at $37^{\circ} \mathrm{C}$ for $35 \mathrm{~min}$ to obtain a cell suspension. Then the suspension was passed through a $40 \mu \mathrm{m}$ cell strainer to remove debris and ensure a single-cell suspension. After a $10 \mathrm{~min}$ centrifugation at $300 \mathrm{~g}$, the pellet was gently resuspended with ice-cold PBS + $0.5 \%$ BSA buffer and incubate for $15 \mathrm{~min}$ at $4^{\circ} \mathrm{C}$ with Myelin Removal Beads II (Miltenyi Biotec). After washing with PBS $+0.5 \% \mathrm{BSA}$ and 10 min centrifugation at $300 \mathrm{~g}$, the pellet was resuspended with PBS + $0.5 \%$ BSA. The single-cell suspension was applied to a column placed on a magnetic stand (LS column and magnets from Miltenyi Biotec) to deplete the myelin fragments by magnetic separation and washed with PBS $+0.5 \%$ BSA. Finally, the cells were centrifuged for $5 \mathrm{~min}$ at $300 \mathrm{~g}$ and the pellet was resuspended with $1 \mathrm{~mL}$ of PBS $+0.5 \%$ BSA. After obtaining a myelin-free single-cell suspension, the microglia were labeled with anti-CD11b immunomagnetic beads and isolated using magnetic columns 
(Miltenyi Biotec). To isolate the neurons, the cell suspension depleted of microglia cells was incubated with a cocktail of antibodies from the Neuron Isolation Kit (Miltenyi Biotec). Non-neuronal cells like astrocytes, oligodendrocytes, endothelial cells or fibroblasts were magnetically labeled and depleted through the magnetic columns; therefore, neurons were isolated by negative selection. To confirm the purity of isolated microglia, we performed RT-qPCR with P2ry 12 and Tmem119, microglia-specific markers. To further ensure microglia and neuron purity when using MACS, following sequencing and alignment, all genes with low expression (average raw read count < 30.9) were removed from further analysis.

\section{Fluorescence-activated cell sorting (FACS) and flow cytometry}

For the $\mathrm{C} \times 3 \mathrm{Cr} 1^{\text {GFP }}$ mice infused with HiLyte ${ }^{\mathrm{TM}}$ Fluor 555 -labeled $\mathrm{A} \beta$, microglia were isolated utilizing FACS. After removing the cerebellum, subcortical area and olfactory bulbs, cortical tissue within $1 \mathrm{~mm}$ of either side of the infusion site was processed into a single-cell suspension using the Neural Tissue Dissociation kit followed by the Myelin Removal Beads II (Miltenyi Biotec) as described above. For WT and TREM2 ${ }^{\text {ko }}$ mice, after the myelin removal steps the pellet were resuspended with $100 \mu \mathrm{l}$ of PBS and the microglia cells were labeled with $5 \mu$ of anti-mouse/human CD11b antibody (clone M1/70) conjugated with Alexa Fluor $^{\mathrm{o}} 647$ (BioLegend) for $20 \mathrm{~min}$ on ice. All cells were then washed and prepared with $1 \mathrm{~mL}$ of PBS + $0.5 \%$ BSA for FACS sorting.

A bio-contained BD FACSAria ${ }^{\text {TM }}$ III sorter (BD Biosciences) was used to sort microglia cells for the two experimental populations. First, live cells were separated from the debris according to their forward scatter and side scatter properties and a second gate was used on individual cells only. The GFP fluorescence was detected with a $525 \mathrm{~nm}$ filter (488 nm laser) for the microglia isolated from the Cx3cr1 ${ }^{\text {GFP }}$ mice, the CD11b/APC fluorescence was collected with a $668 \mathrm{~nm}$ filter ( $647 \mathrm{~nm}$ laser) for the WT and TREM $2{ }^{\mathrm{ko}}$ mice and the 555 -labeled $A \beta$ fluorescence was detected with a $613 \mathrm{~nm}$ filter $(555 \mathrm{~nm}$ laser). 2,000 microglia with high GFP signal but low 555-labeled A $\beta$ signal, were considered unexposed to $\mathrm{Ab}$ and termed single-positive cells (single+). 2,000 microglia cells with a high signal for both labels (GFP and 555-labeled $\mathrm{Ab}$ ) were considered exposed to $\mathrm{Ab}$ and termed dual-positive cells (dual+). The single+ and dual+ microglial populations were sorted into $1.5 \mathrm{~mL}$ tubes containing $350 \mu \mathrm{L}$ of RLT lysis buffer from the RNeasy Micro kit (Qiagen) and 3.5 $\mathrm{LL}$ of 2-Mercaptoethanol. After the FACS sorting, the biocontained BD FACSAria ${ }^{\text {TM }}$ III sorter was used with the same gates to count and estimate the percentages of the total microglia population and single+ and dual+ microglia population in a total of $1,000,000$ live cells.

\section{RNA isolation and RNA-sequencing}


RNA was isolated from the MACS-isolated microglia and neurons and FACS single+ and dual+ microglia following the manufacturer instructions for the RNeasy Mini kit and RNeasy Micro kit (Qiagen) respectively. After RNA isolation, concentration was measured with a Qubit 3.0 Fluorometer (Thermofisher) before quality assessment with the 2100 Bioanalyzer instrument (Agilent Technologies). Sequencing libraries for MACS-isolated cells were generated using mRNA Library Prep Reagent set (Illumina) as previously described ${ }^{49}$. The total RNA from sorted cells was fragmented and converted into CDNA using the SMART-Seq ${ }^{\circledR}$ v4 Ultra ${ }^{\circledR}$ Low Input RNA Kit for Sequencing (Takara Bio). Briefly, a minimum of $10 \mathrm{pg}$ of purified total RNA was used to perform a first-strand cDNA synthesis in a PCR clean workstation. After amplification by LD PCR and purification using the Agencourt AMPure XP beads (Beckman Coulter), $1 \mu \mathrm{L}$ of the amplified cDNA was used for validation using the Agilent 2100 Bioanalyzer on a High Sensitivity DNA chip. $1 \mathrm{ng}$ of the cDNA was used with the Nextera DNA Library Preparation Kit (Illumina) to prepare the libraries. After tagmentation, the cDNA was linked with two different index adapters from the Nextera XT Index Kit (Illumina) during the amplification by PCR. Each sample received a unique combination of two specific index/barcodes during the library generation. Finally, the libraries were purified using Agencourt AMPure XP beads that provide a size selection to remove short library fragments. Again, $1 \mu \mathrm{L}$ of the libraries was used for validation using the Agilent 2100 Bioanalyzer on a High Sensitivity DNA chip. After preparation of the libraries, sequencing was performed by the Next Generation Sequencing Center (University of Pennsylvania, https://ngsc.med.upenn.edu/) on HiSeq 2500 machine.

\section{mRNA-seq data processing}

Following initial processing and quality control, the sequencing data was aligned to the mouse genome mm10 using Subread/featureCounts (v1.5.3; https://sourceforge.net/projects/subread/files/subread$1.5 .3 /$ ) with an average read depth of $21,554,242$ successfully aligned reads. Statistical analysis was carried out using Rsubread (v1.34.2;

https://bioconductor.org/packages/release/bioc/html/Rsubread.html), DEseq2 (1.24.0; https://bioconductor.org/packages/release/bioc/html/DESeq2.html), and edgeR (v3.26.5; https://bioconductor.org/packages/release/bioc/html/edgeR.html), all in the R environment (v3.6.0; https://www.r-project.org/). Functional annotation clustering was performed using the Database for Annotation, Visualization and Integrated Discovery (DAVID v6.8, https://david.ncifcrf.gov). All GO terms were considered significant if $p<0.05$ following corrections for multiple comparisons using the BenjaminiHochberg method to control the FDR. t-Distributed Stochastic Neighbor Embedding (t-SNE) plots were generated to visualize patterns of similarity between groups. To generate the t-SNE, normalized data from all genes in the analysis were submitted and the perplexity set as high as possible given the total number of samples in the analysis with the theta set to 0.5 .

\section{Single-cell isolation and library creation}


Separate biological replicates from $A \beta E 3$ or $A \beta E 4$ infused WT and Trem2 ${ }^{\mathrm{ko}}$ mice were used for single cell RNA-seq (scRNA-seq) and infused as described above. $24 \mathrm{~h}$ after the injections mice were perfused with PBS. After the brain extraction, cortical tissue around the injection site were excised using adult mouse brain matrix at $1 \mathrm{~mm}$ before and after the injection site. The brain tissue was then dissociated according to manufacturer instructions (Miltenyi Biotec) as described above. CD11b-purified cells were resuspended in $0.04 \%$ BSA/PBS solution and passed through a $40-\mu \mathrm{m}$ nylon and a $20-\mu \mathrm{m}$ nylon cell strainer to obtain single cell suspension. The cell viability and number were assessed using an Countess II FL Automated Cell Counter (ThermoFisher) with Live/Dead viability/Cytotoxicity kit (Invitrogen). The single cell suspension with $>90 \%$ viability was immediately loaded onto the Chromium controller (10x Genomics) in order to capture $\sim 5,000$ cells per sample. Libraries were generated with Chromium single cell 3' chip kit v3 for scRNA-seq (10x Genomics) according to the manufacturer's instructions and then the quality assessed using Agilent Bioanalyzer 2100. Sequencing was performed using NovaSeq (Illumina) by MedGenome Inc. with a custom sequencing setting (28bp for Read1 and $91 \mathrm{bp}$ for Read2) to obtain an average sequencing depth of $45 \mathrm{~K}$ reads/cell.

\section{scRNA-seq data processing}

After libraries were sequenced and quality control was performed, samples were aligned to the $\mathrm{mm} 10$ mouse reference genome using the Cell Ranger 3.0.1 pipeline (https://support.10xgenomics.com/singlecell-gene-expression/software/pipelines/latest/what-is-cell-ranger). Each sample was then aggregated using the cellranger aggr function to produce a raw UMI count matrix containing the number of reads for genes in each cell per sample. The expression matrix was then loaded into $\mathrm{R}$ for further analysis and visualization using Seurat v3.0.2 ${ }^{50}$. Genes expressed in fewer than 200 cells were excluded from further analysis. The number of genes expressed in each cell ( $n G e n e$ ), the number of reads in each cell (nCount) and the percentage of reads mapped to mitochondrial genes (percent.mito) were obtained for each cell. Cells were then filtered to reduce the potential of including doublet and low-quality cells using the following criteria: $200<\mathrm{nGene}<8500 ; 500<\mathrm{nCount}<90000$; and percent.mito $<25 \%$. Feature counts were normalized using LogNormalize method with a scale factor of 10000; and the effects of nGene, nCount and percent.mito were regressed out using the ScaleData method. A shared nearest neighbor (SNN) graph was constructed using FindNeighbors function with default parameters. Using the Louvain algorithm implemented in FindClusters function with a clustering resolution of 0.2 and the first 50 PCs, we identified 16 clusters. To determine the cell types, present in each cluster, we examined the expression levels of cell type specific markers across each cluster and identified clusters containing unique populations of microglia, neurons, astrocytes, vascular cells, ependymal cells and myeloid cells. We used FindAllMarkers function to identify genes that act as markers for each cluster, using the Wilcoxon ranksum test. A gene was considered the marker of a cluster if it had a Bonferroni-adjusted $p$-value $<0.01$ and an average log fold change $>0.1$. The data were then filtered to contain clusters containing only microglia and cells with a normalized expression < 1 for Aldoc, F13a1, Mylk, Meg3, Gfap, Thy1, Slc17a7, Olfm1, Aqp4, DIx1, Olig2, Foxj1, Nkg7, Vtn, Flt1, Acta2 and S100a9 were removed, resulting in the retention of

Page 22/39 
36,244 microglial cells. To control for the potential of batch effect we regressed out the percentage of UMI counts in each cell belonging to several sex-linked genes: Ddx3y, Eif2s3y, Xist, Kdm5d and Uty. Using a clustering resolution of 0.1 and the first 50 PCs, we identified 5 separate clusters. To perform differential expression analysis, we used Seurat's FindMarkers function and performed Wilcoxon ranksum tests. A gene was considered differentially expressed if it had a Bonferroni-corrected $p$-value $<0.05$ and a natural $\log$ fold change $(\log F C)>0.1$. Seurat data objects were reformatted for pseudotime analysis using the SingleCellExperiment package and trajectory analysis was performed using Slingshot ${ }^{51}$ establishing cluster 1 a as the starting cluster and clusters 2,3 and 4 as terminal clusters.

\section{Fluorescence in situ hybridization (FISH)}

In a separate infused cohort, mice were perfused, tissue fixed and sectioned as documented above. RNAscope experiments were performed using the Multiplex Fluorescent Reagent kit v2 (Advanced Cell Diagnostics) following the manufacturer's recommendations with minor adjustments. Six freshly sectioned tissues per animal were mounted onto superfrost plus slides within a $0.75^{\prime \prime} \times 0.75^{\prime \prime}$ square and baked at $60^{\circ} \mathrm{C}$ for $60 \mathrm{~min}$. Slides were dehydrated using a series of ethanol dilution steps, then submerged in target retrieval reagent at $100^{\circ} \mathrm{C}$ for $10 \mathrm{~min}$. Protease digestion was performed at $40^{\circ} \mathrm{C}$ for 30 min using Protease III and probe hybridization was carried out at $40^{\circ} \mathrm{C}$ for $2 \mathrm{~h}$. We used probe sets available from ACD for Adgre1 and Tmem119. Following the amplification steps, the sections were counterstained with DAPI and coverslipped. Imaging was carried out using a Nikon Eclipse 90i microscope at 20X magnification with tiled imaging of the entire infusion site and analyzed using NIS Elements software (Nikon Instruments Inc.). Four $200 \mu \mathrm{m}$ x $200 \mu \mathrm{m}$ ROls were drawn and a threshold established for each probe to determine the area of puncta coverage. The $4 \mathrm{ROI}$ were averaged together to create one data point per infusion site representing the average area of puncta coverage within $200 \mu \mathrm{m}$ of the edge of the infusion site.

\section{Two-photon imaging and statistical analysis}

We used $\mathrm{C} \times 3 \mathrm{cr}^{\mathrm{GFP}}$ male mice (3 mo.) to determine the microglial response of $\mathrm{A} \beta \mathrm{E} 3$ or A $\mathrm{AE} 4$ utilizing twophoton imaging ${ }^{52}$. Briefly, the animals were anesthetized I.P. with a cocktail of ketamine/xylazine (75/10 $\mathrm{mg} / \mathrm{kg}$ ) and placed in a stereotaxic frame. Body temperature was maintained at $37.6^{\circ} \mathrm{C}$. The skin above the skull was removed and a well of $2 \mathrm{~cm}$ diameter was built over both hemispheres with a light-curable cement (Composite Flowable) to hold a saline immersion for the microscope objective. The craniotomy was conducted with a high-speed dental drill over the parietal cortex bilaterally. The craniotomy site was regularly flushed with saline to wash out bone fragments and prevent thermal damage. The exposed cortex was injected with Hi-Lyte ${ }^{T M}$ Fluor 555-labeled A $\beta$ (Anaspec) combined with native E3 or E4 particles pre-incubated for $24 \mathrm{~h}$. The injection was performed at an approximate depth of $300 \mu \mathrm{m}$ below the surface of the cortex with a borosilicate glass capillary tube (WPI) pulled with a two-stage vertical pipette puller (Narishige) to a tip diameter of approximately $10 \mu \mathrm{m}$ and coupled with flexible tubing to a 
picospritzer microinjection dispense system (Parker). Mice were randomly assigned to either the ABE3 or $A \beta E 4$ group. After the injection, the mice were moved under the two-photon microscope within 5 minutes and anesthesia was maintained with $40 \mathrm{mg} / \mathrm{ml} / \mathrm{h}$ ketamine.

In vivo imaging was conducted with an Ultima IV two-photon laser scanning microscope (Bruker) with an InSight DS Laser system (Spectra-Physics) tuned at a wavelength of $920 \mathrm{~nm}$. Two channels were collected red (595/50) and green $(525 / 50 \mathrm{~nm})$ to visualize the 555-labeled $A \beta$ and microglia, respectively. Images were collected with 16x Nikon objective lens on Prairie View software using a time series of 11 slices with $1024 \times 1024$ matrix size, dwell time 4.4 microseconds and $3 \mu \mathrm{m}$ step size. The effective inplane resolution was $0.4 \mu \mathrm{m}$ per pixel. The Z-stack images were collected in Galvo acquisition mode such that each Z-stack was collected every minute for a total duration of $80 \mathrm{~min}$.

For each animal, Z-stack images were analyzed to quantify the microglial approach and microglial injection site coverage over time. Initially, the data was loaded in ImageJ (National Institutes of Health) and a rigid-body co-registration with the StackReg plugin was used to account for global temporal drifts of the field of view. The Z-stacks were transformed to sum-based XY projections by linear interpolation using the 3D Project function, allowing for time series analysis of specific regions of interest. We generated the panels illustrating cell displacement from those datasets by assigning red to the beginning of the time series $(\mathrm{t}=0)$ and green to subsequent time points (10 $\mathrm{min}$ or $80 \mathrm{~min})$. Then, the two colorcoded images were merged in ImageJ to reveal spatial details, with red showing the original location, green for the new cellular location after a particular segment of time has elapsed (0-10 min or 0-80 min) and yellow at the regions where the two channels overlap. The 555-labeled $A \beta$ infusion site was shown in white to display the increasing coverage as cell processes approach. The time series were also saved as movies presented in the supplementary material.

The datasets were then converted to mat structures and all subsequent analyses were performed in MATLAB (Math Works), using standard image processing and statistical functions. Briefly, the red channel showing the infusion site was thresholded using the Otsu's method with the multithresh Matlab function. The resulting images were binarized and small features outside of the infusion site were removed using the bwareaopen function. The mask was then dilated to smooth the borders and account for the lower contrast of the diffusion gradient around the borders. The percent coverage by the microglial processes over time was then computed as the increase of the green channel intensity above 1.5 times the standard deviation baseline of the green channel within the binary mask.

The mean distance of the cell processes to the infusion site over time was computed by eroding the binary mask described above and computing the Euclidean distance transformation of the full binary image using the bwdist function. Then, each pixel in the green channel over 1.5 standard deviations of the intensity was mapped with that transformation and assigned a number that is the distance between that pixel and the edge of the infusion site forming progressive concentric level sets with increasing proximity to the infusion site. The change of this Euclidean distance over time was converted from pixels to microns using the effective in-plane resolution of the imaging data and plotted as a time series. 


\section{Primary microglia cell culture}

Primary microglial culture and $A \beta$ uptake assay were performed using WT, E3, E4, E3/Trem2 ${ }^{\mathrm{ko}}$ and E4/Trem2 ${ }^{\mathrm{ko}}$ pups (1-3 days old) as described before ${ }^{42,43}$. Briefly, the cortices and hippocampi were mechanically dissociated using a sterile Pasteur pipette. The dissociated cells were plated in $75 \mathrm{~cm}^{2}$ flasks with DMEM/F12 medium (Thermo Fisher) containing 10\% FBS at DIV0. $24 \mathrm{~h}$ after plating (DIV1), the media was replaced to remove debris. Microglia were collected by tapping at DIV14 and plated on $0.01 \%$ poly-L-lysine (Sigma) coated $12 \mathrm{~mm}$ circular coverslips in 24-well plates with the density of 60,000 cells/well. $24 \mathrm{~h}$ after plating, cells in the treatment groups were treated with $1 \mu \mathrm{M} \mathrm{Hi-Lyte}{ }^{\mathrm{TM}}$ Fluor 488 labeled $A \beta$ (Anaspec) at $37^{\circ} \mathrm{C}$ for $1 \mathrm{~h}$. Following the treatment, cells were washed three times with PBS, fixed in $4 \%$ PFA and permeabilized with $0.2 \%$ Triton X-100 at room temperature. Microglia were then labeled with Anti-IBA1 antibody $(1: 500)$ at $4{ }^{\circ} \mathrm{C}$ for $18 \mathrm{~h}$ and followed by $2 \mathrm{~h}$ incubation with horse antirabbit 594 secondary antibody and stained with DAPI to visualize nuclei.

Fluorescent images of in vitro Ab-treated microglia were taken on a Nikon Eclipse 90i microscope (20X magnification) and analyzed in NIS elements (Nikon Instruments Inc.). Exposure levels for each channel were consistent across all genotypes and samples. Images were thresholded to identify microglia, nuclei and $A b$. To assess the $A \beta$ uptake in microglia, the percentage of microglia containing Ab ((IBA1+ colocalized with 488-labeled $A \beta+)$ / (IBA1+ and $488 A \beta-)$ ) was calculated and averaged across all images in each genotype. To assess the morphology of the microglia, the circularity of thresholded IBA1+ microglia was calculated and averaged across all images in each genotype on a scale of 0 to 1 , with 1 being a perfect circle.

\section{QUANTIFICATION AND STATISTICAL ANALYSIS}

Sample sizes ( $\mathrm{n}$ ) indicated in the figure legend 1 indicate number of separate measurements. Sample sizes ( $n$ ) indicated in the figure legend 2-7 correspond to the number of biological replicates analyzed. All researchers were blinded to experimental groups during the analysis. All results are reported as means \pm SEM.

Unless otherwise indicated, all statistical analyses were performed in GraphPad Prism (v 8.2.0), or in R ( $\mathrm{v}$ 3.6.0) and significance was determined as $p<0.05$. Number of experiments and statistical information are stated in the corresponding figure legends. In figures, asterisks denote statistical significance marked by * $p<0.05 ; * \star p<0.01 ; * \star \star x<0.001$.

\section{Declarations}

Acknowledgements: NA

Author Contributions: 
Conceptualization, N.F.F., K.N.N., C.M.W., I.L., and R.K.;

Methodology, N.F.F., K.N.N., R.K., I.L.;

Validation, N.F.F., K.N.N., C.M.W., B.E.P., B.E.I., V.E.K., Y.Y.T., X.H., R.J.B., R.K., I.L.;

Formal analysis, N.F.F., C.M.W., K.N.N., B.E.I. V.E.K., Y.Y.T., X.H.;

Resources, N.F.F., K.N.N., C.M.W., R.K., I.L.;

Data curation, C.M.W., N.F.F., K.N.N. B.E.I. V.E.K., Y.Y.T., X.H.;

writing-original draft preparation, N.F.F., K.N.N., C.M.W.;

writing-review and editing, N.F.F., K.N.N., C.M.W., R.J.B., B.E.I., V.E.K., Y.Y.T., X.H., R.K., I.L.;

Visualization, C.M.W., K.N.N., N.F.F. R.K., I.L. B.E.I.;

Supervision, N.F.F., K.N.N., R.K., I.L.;

Project administration, N.F.F., R.K., I.L.;

Funding acquisition, N.F.F., R.K., I.L.

All authors read and approved the final manuscript.

Competing Interests statement: The authors declare no competing interests.

\section{References}

1. Association A. 2019 ALZHEIMER'S DISEASE FACTS AND FIGURES. (2019).

2. Corder EH, et al. Gene dose of apolipoprotein E type 4 allele and the risk of Alzheimer's disease in late onset families. Science261, 921-923 (1993).

3. Saunders AM, et al. Association of apolipoprotein E allele epsilon 4 with late-onset familial and sporadic Alzheimer's disease. Neurology43, 1467-1472 (1993).

4. Weisgraber $\mathrm{KH}$. Apolipoprotein E distribution among human plasma lipoproteins: role of the cysteinearginine interchange at residue 112. Journal of lipid research31, 1503-1511 (1990).

5. Hatters DM, Peters-Libeu CA, Weisgraber KH. Apolipoprotein E structure: insights into function. Trends Biochem Sci31, 445-454 (2006).

6. Lefterov I, et al. APOE2 orchestrated differences in transcriptomic and lipidomic profiles of postmortem AD brain. Alzheimers Res Ther11, 113-113 (2019). 
7. Wolfe CM, Fitz NF, Nam KN, Lefterov I, Koldamova R. The Role of APOE and TREM2 in Alzheimer's Disease-Current Understanding and Perspectives. International journal of molecular sciences20, (2018).

8. Koldamova R, Fitz NF, Lefterov I. ATP-binding cassette transporter A1: from metabolism to neurodegeneration. Neurobiol Dis72 Pt A, 13-21 (2014).

9. DeMattos RB, et al. Purification and characterization of astrocyte-secreted apolipoprotein E and Jcontaining lipoproteins from wild-type and human apoE transgenic mice. Neurochem Int39, 415-425 (2001).

10. LaDu MJ, Falduto MT, Manelli AM, Reardon CA, Getz GS, Frail DE. Isoform-specific binding of apolipoprotein E to beta-amyloid. J Biol Chem269, 23403-23406 (1994).

11. Deane R, et al. apoE isoform-specific disruption of amyloid beta peptide clearance from mouse brain. J Clin Invest118, 4002-4013 (2008).

12. Jiang Q, et al. ApoE promotes the proteolytic degradation of Abeta. Neuron58, 681-693 (2008).

13. Fitz NF, et al. Abca1 deficiency affects Alzheimer's disease-like phenotype in human ApoE4 but not in ApoE3-targeted replacement mice. The Journal of neuroscience : the official journal of the Society for Neuroscience32, 13125-13136 (2012).

14. Holtzman DM, et al. Apolipoprotein $\mathrm{E}$ isoform-dependent amyloid deposition and neuritic degeneration in a mouse model of Alzheimer's disease. Proc Natl Acad Sci U S A97, 2892-2897 (2000).

15. Verghese PB, et al. ApoE influences amyloid-beta (Abeta) clearance despite minimal apoE/Abeta association in physiological conditions. Proc Natl Acad Sci U S A110, E1807-1816 (2013).

16. Hansen DV, Hanson JE, Sheng M. Microglia in Alzheimer's disease. The Journal of cell biology217, 459-472 (2018).

17. Deczkowska A, Keren-Shaul H, Weiner A, Colonna M, Schwartz M, Amit I. Disease-Associated Microglia: A Universal Immune Sensor of Neurodegeneration. Cel/173, 1073-1081 (2018).

18. Selkoe DJ, Hardy J. The amyloid hypothesis of Alzheimer's disease at 25 years. EMBO molecular medicine8, 595-608 (2016).

19. Keren-Shaul H, et al. A Unique Microglia Type Associated with Restricting Development of Alzheimer's Disease. Cel/169, 1276-1290.e1217 (2017).

20. Atagi $Y$, et al. Apolipoprotein E Is a Ligand for Triggering Receptor Expressed on Myeloid Cells 2 (TREM2). J Biol Chem290, 26043-26050 (2015). 
21. Yeh FL, Wang Y, Tom I, Gonzalez LC, Sheng M. TREM2 Binds to Apolipoproteins, Including APOE and CLU/APOJ, and Thereby Facilitates Uptake of Amyloid-Beta by Microglia. Neuron91, 328-340 (2016).

22. Song $\mathrm{W}$, et al. Alzheimer's disease-associated TREM2 variants exhibit either decreased or increased ligand-dependent activation. Alzheimers Dement13, 381-387 (2017).

23. Yuan $P$, et al. TREM2 Haplodeficiency in Mice and Humans Impairs the Microglia Barrier Function Leading to Decreased Amyloid Compaction and Severe Axonal Dystrophy. Neuron90, 724-739 (2016).

24. Fitz NF, et al. Trem2 deficiency differentially affects phenotype and transcriptome of human APOE3 and APOE4 mice. Mol Neurodegener15, 41 (2020).

25. Wahrle SE, et al. ABCA1 is required for normal central nervous system ApoE levels and for lipidation of astrocyte-secreted apoE. J Biol Chem279, 40987-40993 (2004).

26. Duong PT, Weibel GL, Lund-Katz S, Rothblat GH, Phillips MC. Characterization and properties of pre beta-HDL particles formed by ABCA1-mediated cellular lipid efflux to apoA-I. Journal of lipid research49, 1006-1014 (2008).

27. Fitz NF, et al. ABCA1 Deficiency Affects Basal Cognitive Deficits and Dendritic Density in Mice. $J$ Alzheimers Dis56, 1075-1085 (2017).

28. Lesné $\mathrm{S}$, et al. A specific amyloid-beta protein assembly in the brain impairs memory. Nature440, 352-357 (2006).

29. Lefterov I, et al. Apolipoprotein A-I deficiency increases cerebral amyloid angiopathy and cognitive deficits in APP/PS1DeltaE9 mice. J Biol Chem285, 36945-36957 (2010).

30. Lee E, et al. Single-nucleotide polymorphisms are associated with cognitive decline at Alzheimer's disease conversion within mild cognitive impairment patients. Alzheimers Dement (Amst)8, 86-95 (2017).

31. Yu FX, Johnston PA, Sudhof TC, Yin HL. gCap39, a calcium ion- and polyphosphoinositide-regulated actin capping protein. Science250, 1413-1415 (1990).

32. Galatro TF, et al. Transcriptomic analysis of purified human cortical microglia reveals age-associated changes. Nat Neurosci20, 1162-1171 (2017).

33. Hammond TR, et al. Single-Cell RNA Sequencing of Microglia throughout the Mouse Lifespan and in the Injured Brain Reveals Complex Cell-State Changes. Immunity50, 253-271 e256 (2019).

34. Wang Y, et al. TREM2 lipid sensing sustains the microglial response in an Alzheimer's disease model. Cel/160, 1061-1071 (2015). 
35. Chausse B, Kakimoto PA, Kann O. Microglia and lipids: how metabolism controls brain innate immunity. Semin Cell Dev Biol, (2020).

36. Kosicek M, Hecimovic S. Phospholipids and Alzheimer's disease: alterations, mechanisms and potential biomarkers. International journal of molecular sciences14, 1310-1322 (2013).

37. Shi Y, Holtzman DM. Interplay between innate immunity and Alzheimer disease: APOE and TREM2 in the spotlight. Nat Rev Immuno/18, 759-772 (2018).

38. Wisniewski T, Drummond E. APOE-amyloid interaction: Therapeutic targets. Neurobiol Dis138, 104784 (2020).

39. Kanekiyo T, Xu H, Bu G. ApoE and Abeta in Alzheimer's disease: accidental encounters or partners? Neuron81, 740-754 (2014).

40. Song WM, Joshita S, Zhou Y, Ulland TK, Gilfillan S, Colonna M. Humanized TREM2 mice reveal microglia-intrinsic and -extrinsic effects of $\mathrm{R} 47 \mathrm{H}$ polymorphism. The Journal of experimental medicine215, 745-760 (2018).

41. Sala Frigerio C, et al. The Major Risk Factors for Alzheimer's Disease: Age, Sex, and Genes Modulate the Microglia Response to Abeta Plaques. Cell Rep27, 1293-1306 e1296 (2019).

42. Fitz $N F$, et al. Liver $X$ receptor agonist treatment ameliorates amyloid pathology and memory deficits caused by high-fat diet in APP23 mice. The Journal of neuroscience : the official journal of the Society for Neuroscience30, 6862-6872 (2010).

43. Lefterov I, Schug J, Mounier A, Nam KN, Fitz NF, Koldamova R. RNA-sequencing reveals transcriptional up-regulation of Trem2 in response to bexarotene treatment. Neurobiol Dis82, 132-140 (2015).

44. Aviram R, et al. Lipidomics Analyses Reveal Temporal and Spatial Lipid Organization and Uncover Daily Oscillations in Intracellular Organelles. Mol Cel/62, 636-648 (2016).

45. Han X, Yang K, Gross RW. Multi-dimensional mass spectrometry-based shotgun lipidomics and novel strategies for lipidomic analyses. Mass Spectrom Rev31, 134-178 (2012).

46. Nam KN, et al. Effect of high fat diet on phenotype, brain transcriptome and lipidome in Alzheimer's model mice. Sci Rep7, 4307 (2017).

47. Tyurina YY, et al. Characterization of cardiolipins and their oxidation products by LC-MS analysis. Chem Phys Lipids179, 3-10 (2014).

48. Marsh SE, et al. The adaptive immune system restrains Alzheimer's disease pathogenesis by modulating microglial function. Proc Natl Acad Sci U S A113, E1316-1325 (2016). 
49. Nam KN, et al. Integrated approach reveals diet, APOE genotype and sex affect immune response in APP mice. Biochim Biophys Acta Mol Basis Dis1864, 152-161 (2018).

50. Butler A, Hoffman P, Smibert P, Papalexi E, Satija R. Integrating single-cell transcriptomic data across different conditions, technologies, and species. Nat Biotechno/36, 411-420 (2018).

51. Street $\mathrm{K}$, et al. Slingshot: cell lineage and pseudotime inference for single-cell transcriptomics. $B M C$ Genomics19, 477 (2018).

52. Iordanova B, Vazquez A, Kozai TD, Fukuda M, Kim SG. Optogenetic investigation of the variable neurovascular coupling along the interhemispheric circuits. J Cereb Blood Flow Metab38, 627-640 (2018).

\section{Figures}


a
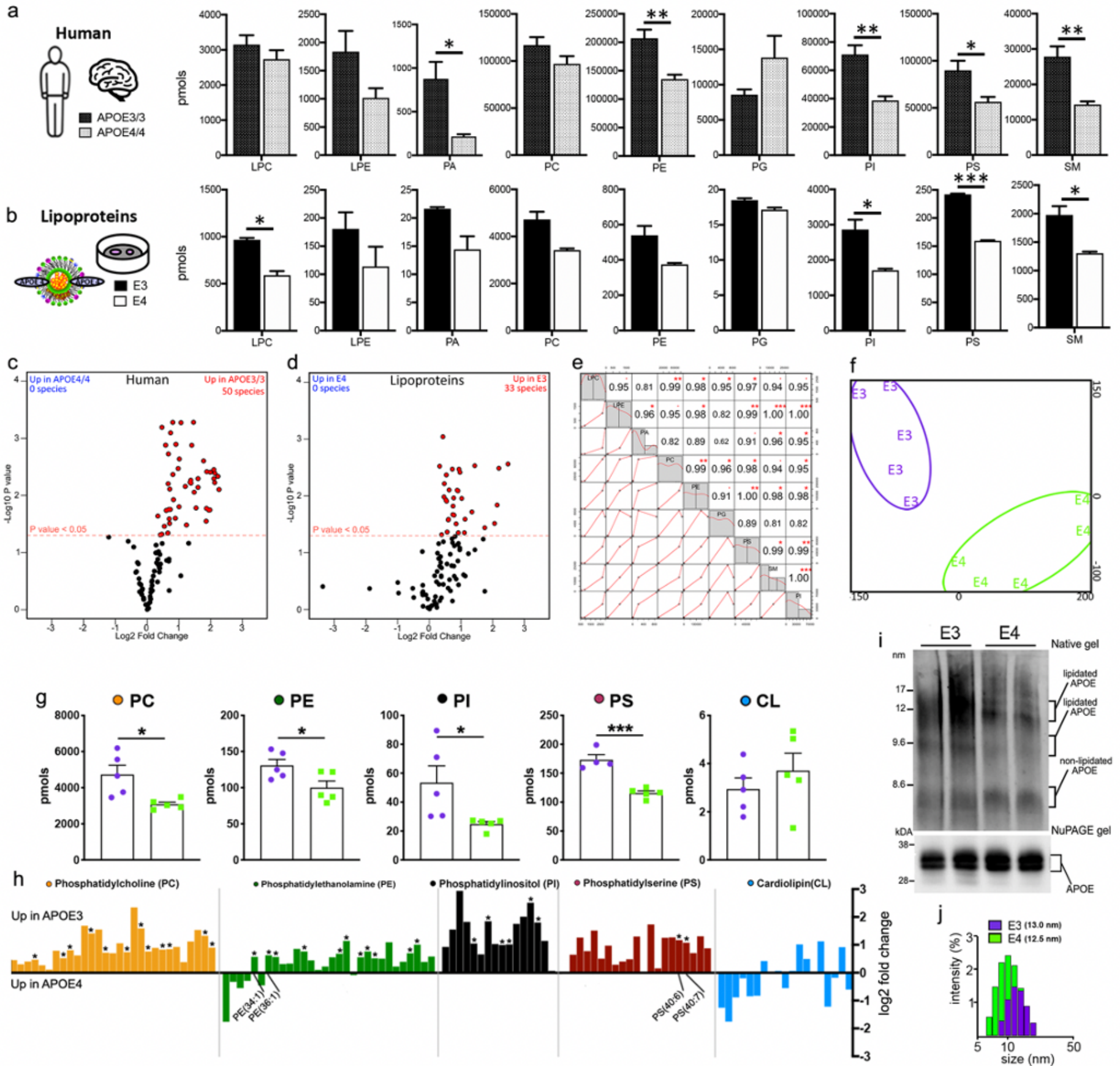

Figure 1

Common phospholipid signature between APOE\&4/4 and APOEع3/3 AD brains and APOE3 and APOE4 native lipoproteins. The phospholipid compositions of APOE $44 / 4$ and APOE $3 / 3$ brains from AD patients and native APOE3 and APOE4 lipoproteins were determined using MDMS-SL. Native lipoproteins were derived from pooled APOE3 or APOE4 astrocyte conditioned media. Brain samples were from the inferior parietal lobe as previously reported. ( $a$ and b) Bar charts of 9 lipid classes analyzed by Shotgun lipidomics in human (a) and native lipoprotein (b) data sets. (c-d) Volcano plots depicting all 103 lipid species commonly identified in the human (c) and lipoprotein (d) datasets. Red denotes species which are significantly upregulated in APOE\&3/3 brains and APOE3 lipoproteins. $X$ axis, log2 fold change and $y$ 
axis, $-\log 10 \mathrm{P}$ value for all lipid species. (e) Correlation matrix of the 9 lipid classes analyzed in human and lipoprotein lipidomics data. (f-h) LC/MS analysis of 5 Phospholipid classes of APOE3 and E4 lipoproteins. (f) tSNE plot shows unique clustering of all lipid from lipoprotein samples according to APOE isoform. (g) Bar charts of major lipid classes of E3 and E4 native lipoproteins comprising all the lipids species shown in panel $h(h)$ Bar chart representing fold change of APOE3 compared to E4 lipoproteins for individual lipid species from five lipid classes. Significantly impacted species which are found in abundance from the PS and PE classes are labeled. (i) Top: Native gel electrophoresis probed for APOE. The diameter of native markers (in $\mathrm{nm}$ ) are shown on the left. The migration of lipidated and nonlipidated APOE is shown on the right indicated with brackets. Bottom: SDS-NuPAGE gel followed by Western blot for APOE. (j) The size distribution of E3 and E4 native lipoproteins was assessed by dynamic light scattering using a Zetasizer. Lipidomic analysis was performed on two independent APOE preparations measured in duplicate. The phospholipid content for each APOE isoform was normalized to APOE protein amount as determined by SDS NuPAGE. Bars represent mean \pm SEM. ${ }^{*} p<0.05$; ${ }^{*} p<0.01$; $\star \star \star ~ p<0.001$ by $t$ test. Cardiolipin (CL); Lysophosphatidylcholine, LPC; Lysophosphatidylethanolamine, LPE; Phosphatidic acid, PA; Phosphatidylcholine, PC; Phosphatidylethanolamine, PE;

Phosphatidylglycerol, PG; Phosphatidylinositol, PI; Phosphatidylserine, PS; and Sphingomyelin, SM. 
a

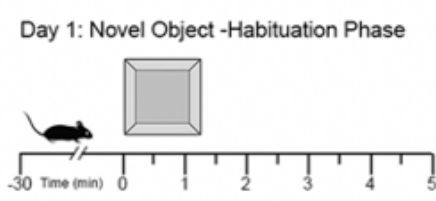

Day 2: Novel Object -Training Phase

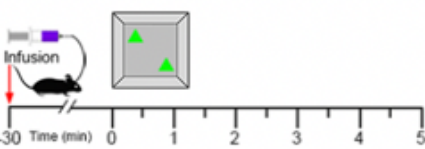

Day 3: Novel Object -Testing Phase
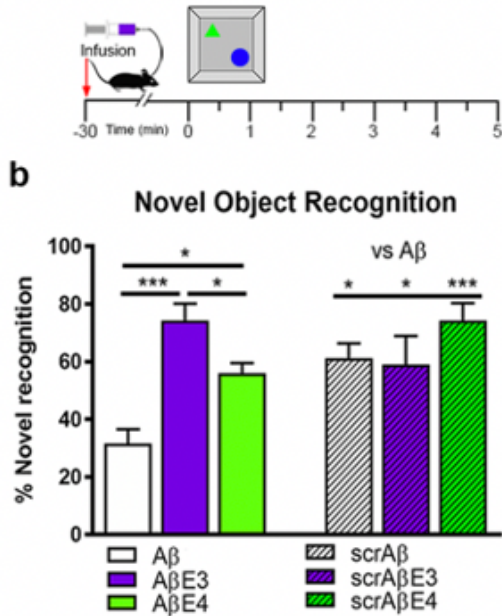

i
C Day 4: Fear Conditioning-Training Phase

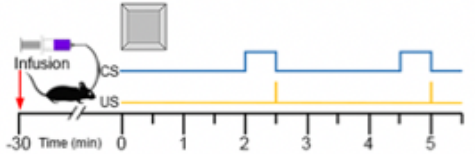

Day 5: Fear Conditioning-Contextual Phase

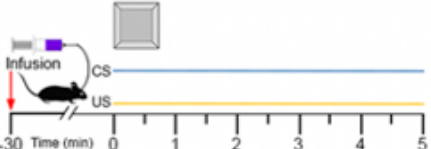

Day 6: Fear Conditioning-Cued Phase

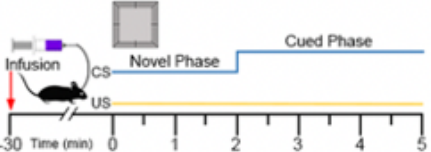

d

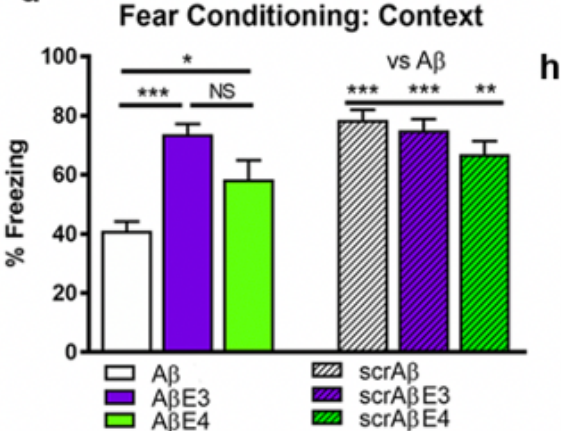

j

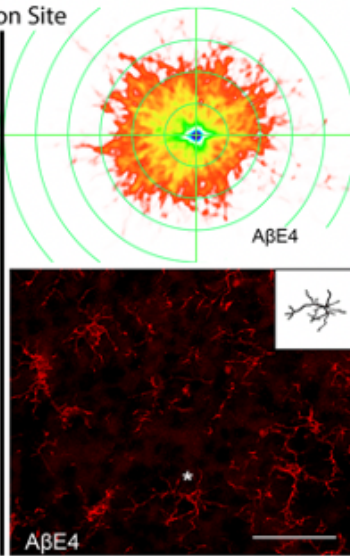

g

h e
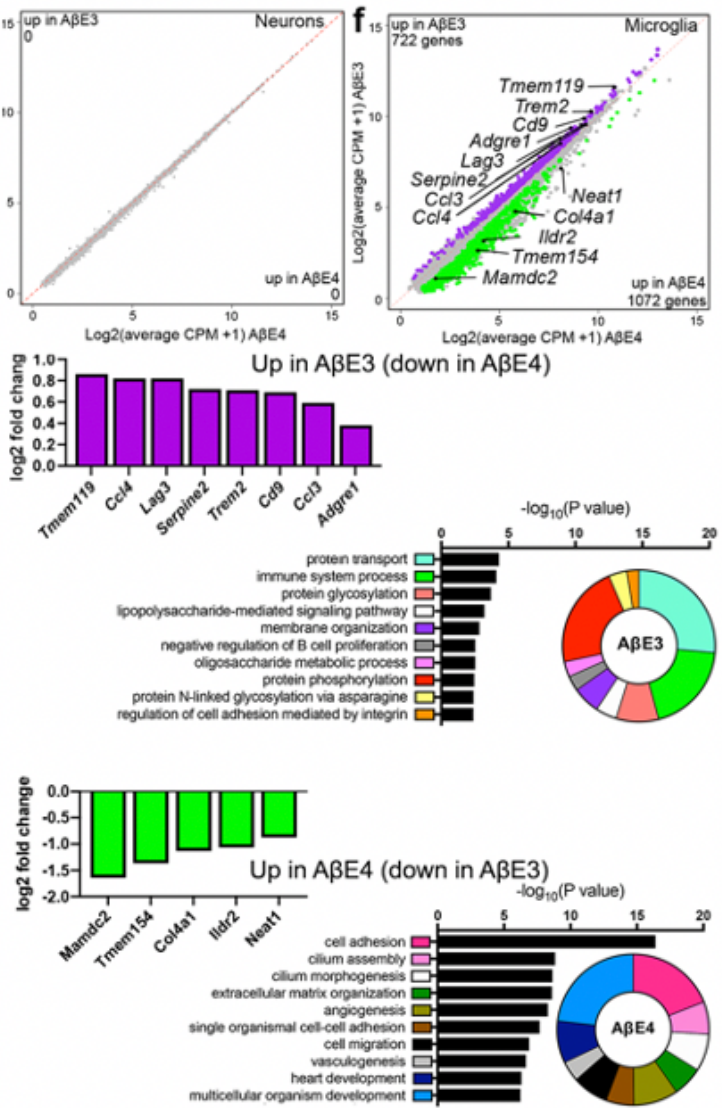

k

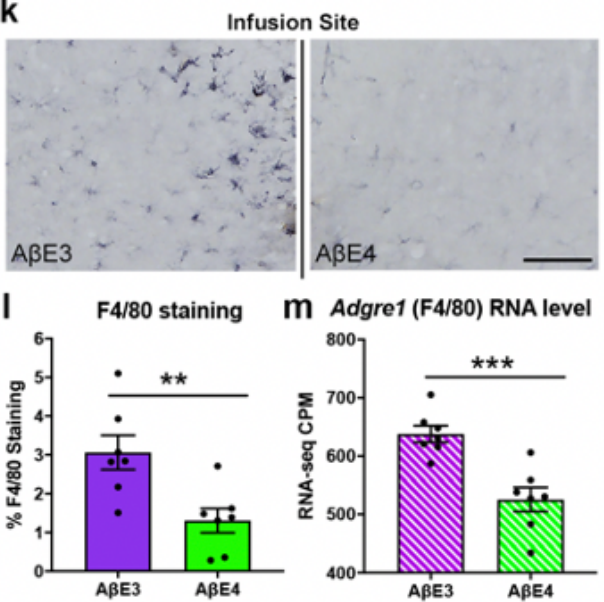

Figure 2

Effect of native E3 and E4 lipoproteins on $A \beta$ induced cognitive decline and microglial response. (a-d) $A \beta$ preparations after the $24 \mathrm{~h}$ incubation $(A \beta, A \beta E 3$ and $A \beta E 4)$, as shown on Suppl. Fig. 2, were infused into the brain of WT mice and cognitive performance examined by novel object recognition $(a-b)$ and fear conditioning (c-d). Scrambled $A \beta$ (scrA $\beta$ ) was used as negative control (scrA $\beta+V$ eh, scrA $\beta E 3$ and scrA $B$ E). All mice were infused 30 min prior to training on days 2 through 6 of behavioral testing as shown on a and $\mathrm{c}$. Analysis by one-way ANOVA followed by Tukeys multiple comparison test. $\mathrm{N}=12$ for $A \beta+/-E 3$ or $E 4$, and $N=8$ for scrA $\beta$ controls. These same $A \beta$ preparations were infused into the cortex of another group of WT mice. $24 \mathrm{~h}$ later microglia and neurons from one cortex were sorted via MACS and 
used for RNA-seq. N=5 mice per group. (e-f) Scatterplots of neuronal (e) and microglial (f) gene expression profile from $A \beta E 3$ vs $A \beta E 4$ injected. Genes significantly upregulated at FDR $<0.05$ in $A \beta E 3$ (purple), and ABE4 (green). ( $g-h)$ Bar plots of significantly upregulated genes and GO terms of $A \beta E 3(\mathrm{~g})$ and $A \beta E 4(h)$ groups. (i-j) IBA1 staining around the infusion site of the same $A \beta E 3$ (i) or ABE4 (j) groups. Imaris filament tracer was used for generation of a microglial projection heatmap around the infusion site. Bottom: representative images of IBA1 staining with Imaris tracing of the labeled $(*)$ microglia as an inset. Scale bar=50 $\mu \mathrm{m}$. N=3 mice/group; 86 microglia/AßE3 and 106 microglia/AßE4. (k-m)

Representative images of the F4/80 (Adgre1) immunostaining around the infusion site of $A \beta E 3$ or $A \beta E 4$ group (k). Images are positioned with the infusion site in the middle. Scale bar=100 $\mu \mathrm{m}$. (I) Bar graph showing percent coverage of $F 4 / 80$ staining increased around the infusion site of the $A \beta E 3$ vs $A \beta E 4$ group. ( $m$ ) Bar graph showing mRNA level of Adgre1 as determined by RNA-seq (CPM, count per million). Analysis by $t$ test. $N=8$ mice per group. Bars represent mean $\pm S E M .{ }^{*} p<0.05, * \star p<0.01 ; * \star \star p<0.001 ; N S$ no significance
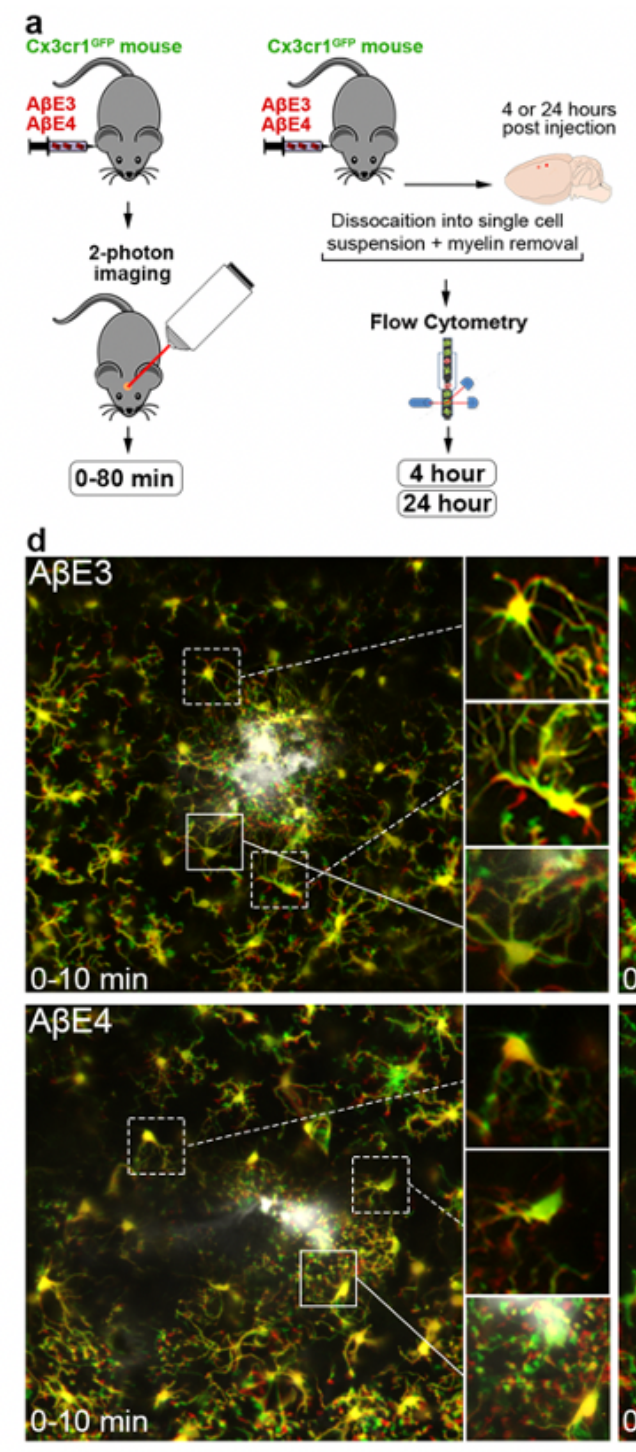
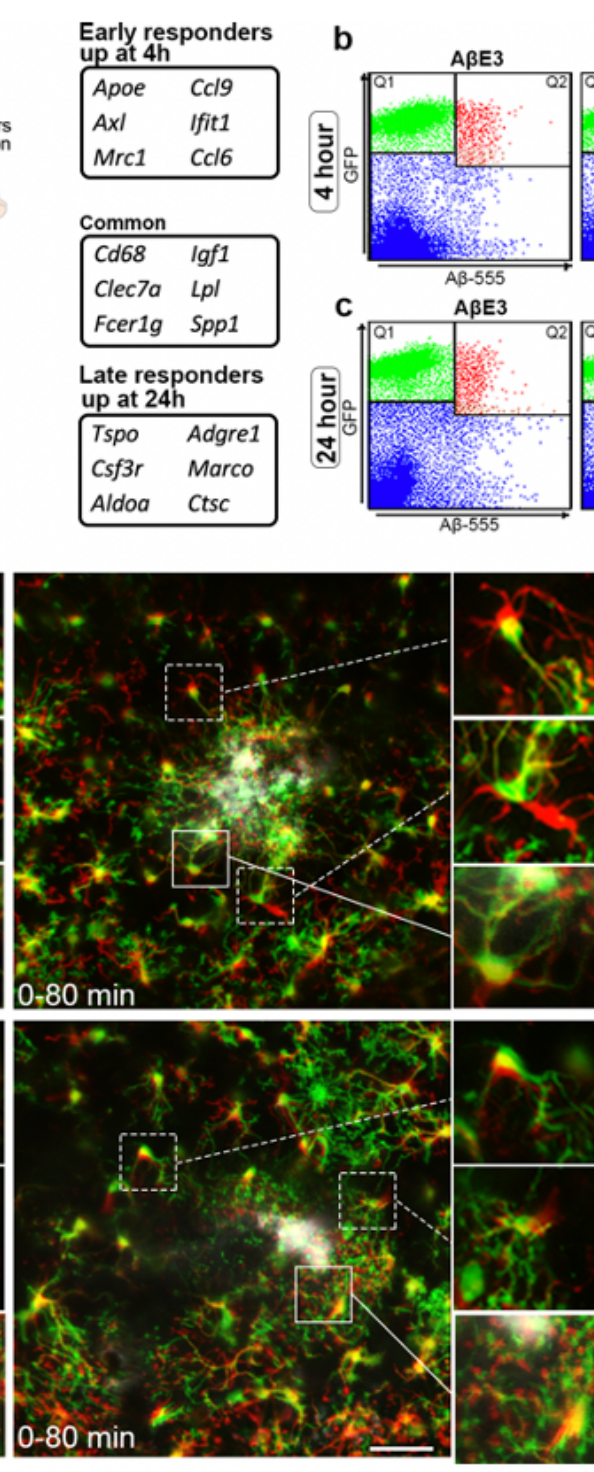
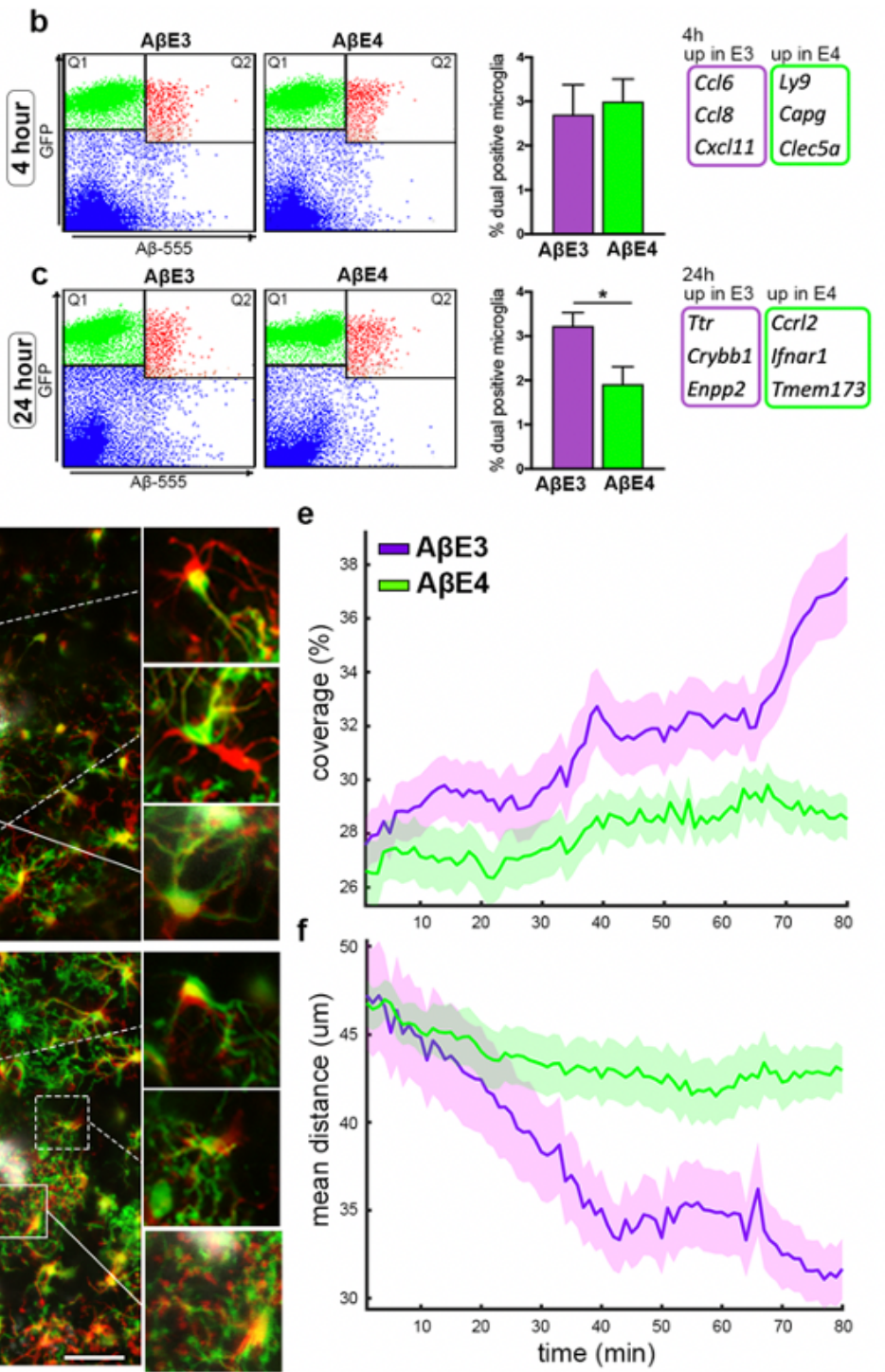

Figure 3 
Microglial interaction with $A \beta$ is affected by APOE isoform. Cx3cr1GFP mice were infused with Hi-Lyte Fluor 555-labeled A $\beta$ pre-incubated with native E3 or E4. (a) Experimental design. (b-c) 4 or $24 \mathrm{~h}$ postinjection the cortex surrounding the injection site was dissociated into a single cell suspension and used for flow cytometry (b-c) and FACS (experiment shown on Fig.4). Flow cytometry scatterplots represent live microglia cells from injected mice. Q1 corresponds to cells that are GFPhigh/Aß-555low and are counted as single+ cells. The Q2 correspond to GFPhigh/A $3-555$ high cells counted as dual+ cells. Flow cytometry scatterplot from $4 \mathrm{~h}$ (b) and $24 \mathrm{~h}$ (c) time points. Bar plots correspond to the percentage of live dual positive microglia cells in $A \beta E 3$ (purple bars) and $A \beta E 4$ groups (green) at $4 \mathrm{~h}$ (b) and $24 \mathrm{~h}$ (c) * ${ }^{*}<0.05$ by t test. $\mathrm{N}=4$ mice/group. (d-f) Two-photon imaging was used to track microglia movement in real time. (d) Representative fields of view from ABE3 (top row) or ABE4 (bottom row) infused mice. Time series are color coded with infused $A \beta$ as white; and initial time point in green and later time points in red as indicated on the panel, (0-10 or 0-80 $\mathrm{min}$ ) with yellow representing the overlap between timepoints. Inserts show details of individual cells demonstrating the extent of migration and directionality of the cellular processes. Scale bar $=50 \mu \mathrm{m}$. (e) Percent coverage of infused $A \beta$ by microglial processes computed from channel overlap. (f) Change of mean distance from microglia to infusion site over time. The standard error within each group is plotted as a bounded line. $\mathrm{N}=4$ mice/group.
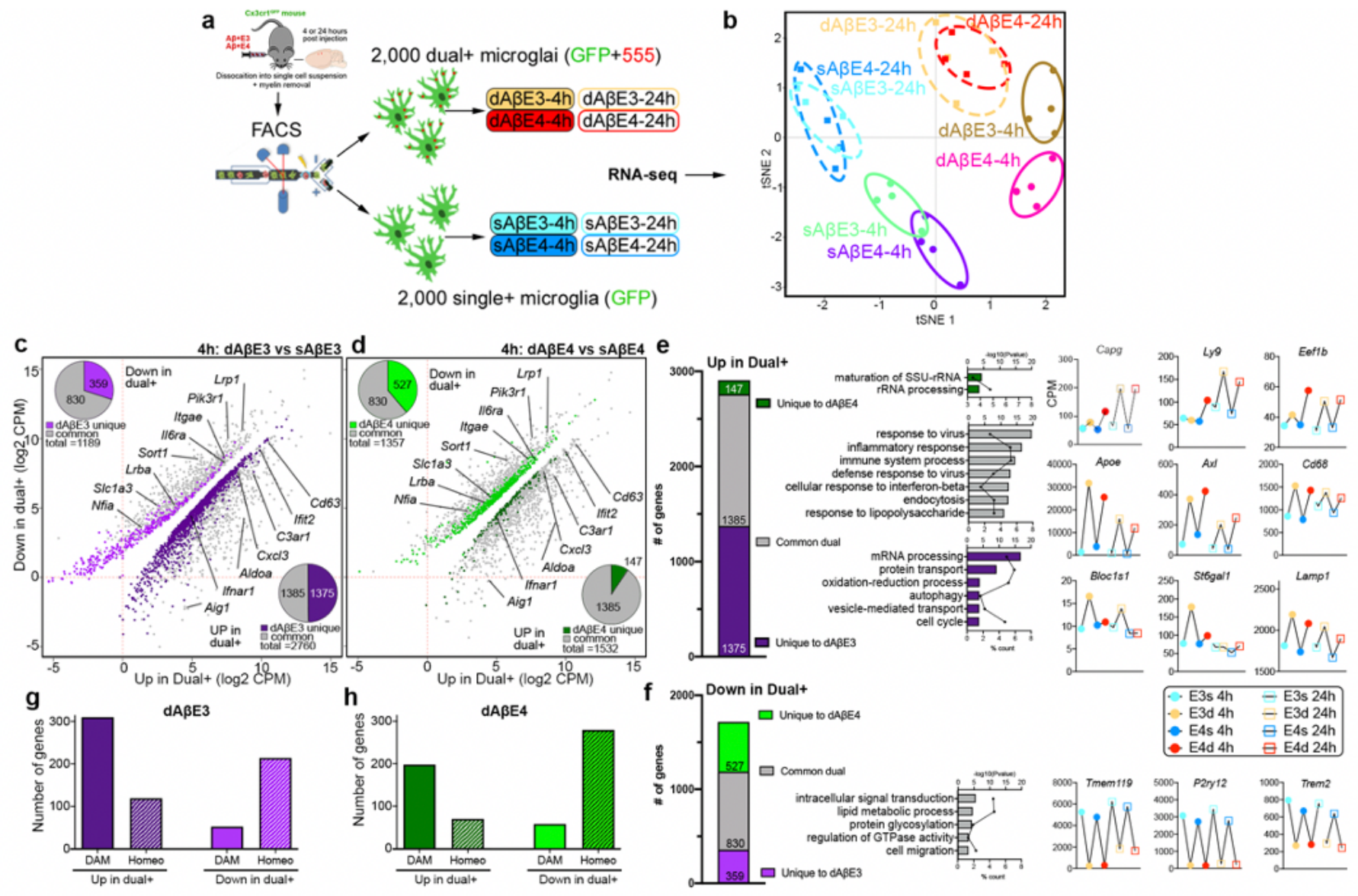

\section{Figure 4}


$A \beta E 3$ and $A \beta E 4$ differentially affect microglia transcriptome. (a) Experimental design: Cx3cr1GFP mice were infused with $A \beta E 3$ or $A \beta E 4$ and processed as shown on Fig. 3 followed by FAC sorting microglia into single+ (referred to as $\mathrm{SA} \beta \mathrm{E} 3$ or $\mathrm{SA} \beta \mathrm{E} 4)$ and dual+ (dABE3 or $\mathrm{dA} \beta \mathrm{E} 4)$ populations and used for RNA-seq. $\mathrm{N}=4$ mice/group. (b) tSNE plot shows clustering of dual+ and single+ microglia for 4 experimental groups. (c-d) Significantly affected transcripts for dual+ vs single+ microglia at $4 \mathrm{~h}$ time point $(\mathrm{FDR}<0.05)$. (c) DEGs in dAßE3 vs sAßE3. (d) DEGs in dAßE4 vs sAßE4. Pie charts in the upper left (down in dual+), and lower right (up in dual+) represent genes common between, or unique to each APOE isoform. (e-f) Proportion of genes, $\mathrm{GO}$ terms and line patterning associated with common or unique genes between dABE3 vs sAßE3 and dAßE4 vs sAßE4. (g) Common and unique upregulated in dual+ vs single+. (g) Common and unique downregulated in dual+ vs single+. For both, bar plots indicate the -log10P value for each term, and the associated point in the center of each bar represents the percent of submitted genes found in each GO term. Line-patterning graphs show example of genes from each category. (g-h) Bar graphs show the number of DAM and homeostatic genes upregulated or downregulated in dual+ for $A \beta E 3$ (g, purple) or AßE4 ( $h$, green) injected mice.

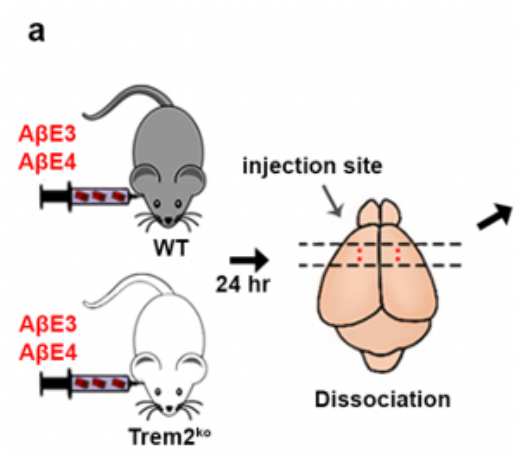

d

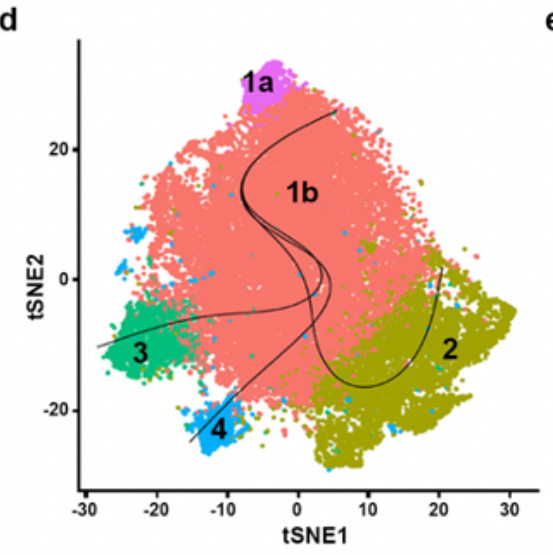

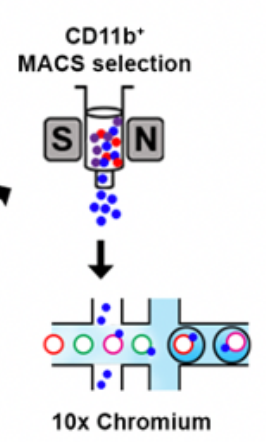

e
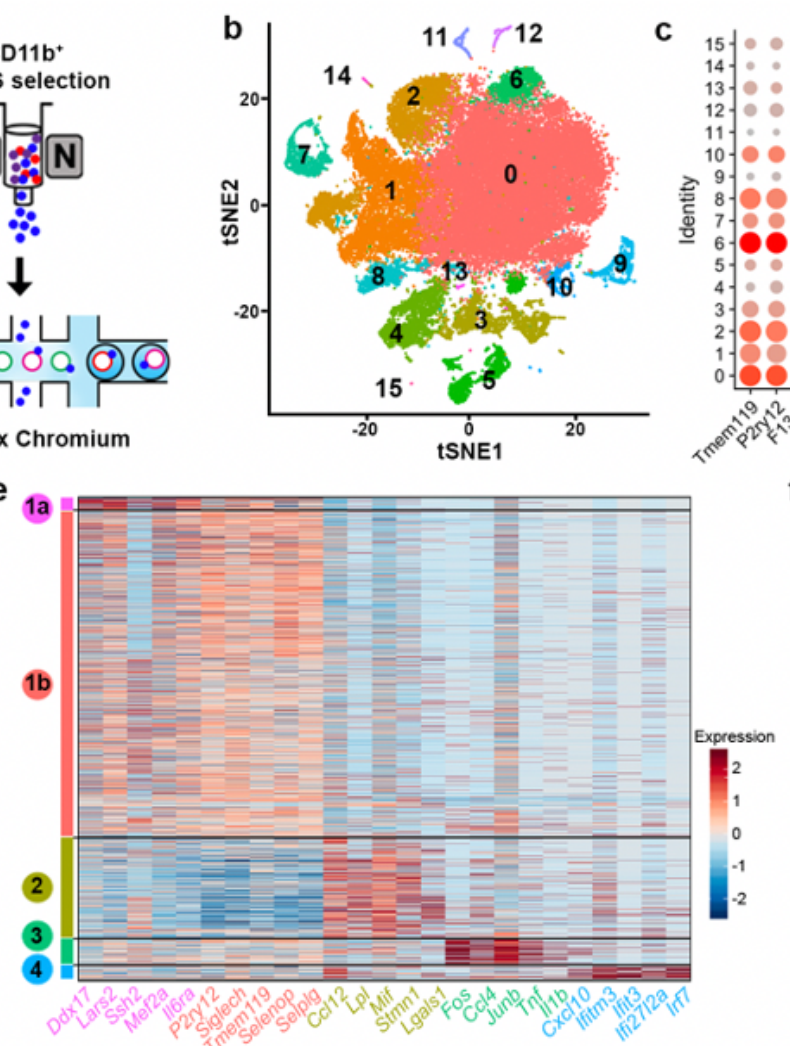

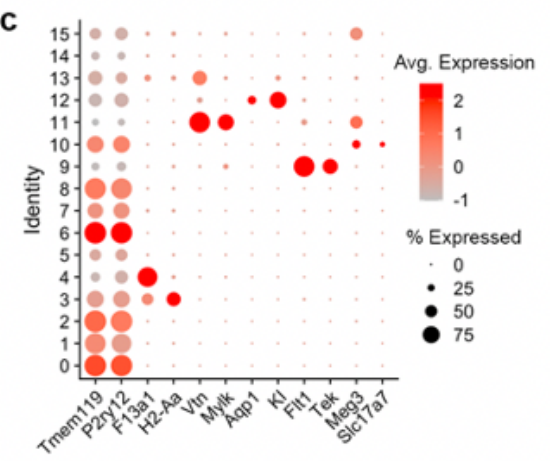

f

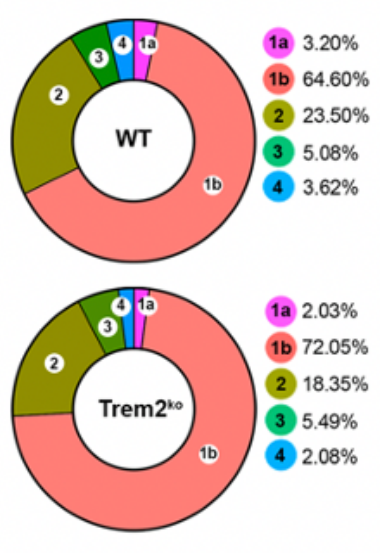

\section{Figure 5}

scRNA-seq data reveal distinct microglial subpopulations in response to $A \beta$ infusion. (a) Schematic workflow of the experimental strategy for scRNA-seq. Microglia from WT and Trem2ko mice injected with $A \beta E 3$ or $A \beta E 4$ sorted using CD11b immunomagnetic beads and immediately applied to the $10 \mathrm{x}$ 


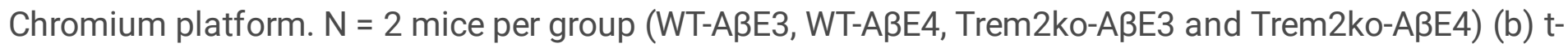
SNE plot showing clusters of 49,817 sequenced cells based on transcriptomic similarities using Seurat. (c) dot plot showing expression of established cell-type specific genes. (d) t-SNE plot showing clusters of 36,244 microglial cells after re-clustering and inferred trajectories by Slingshot. (e) Heatmap showing the expression of top 5 genes in each cluster. (f) Proportion of cells in each cluster from WT and Trem2ko microglia.
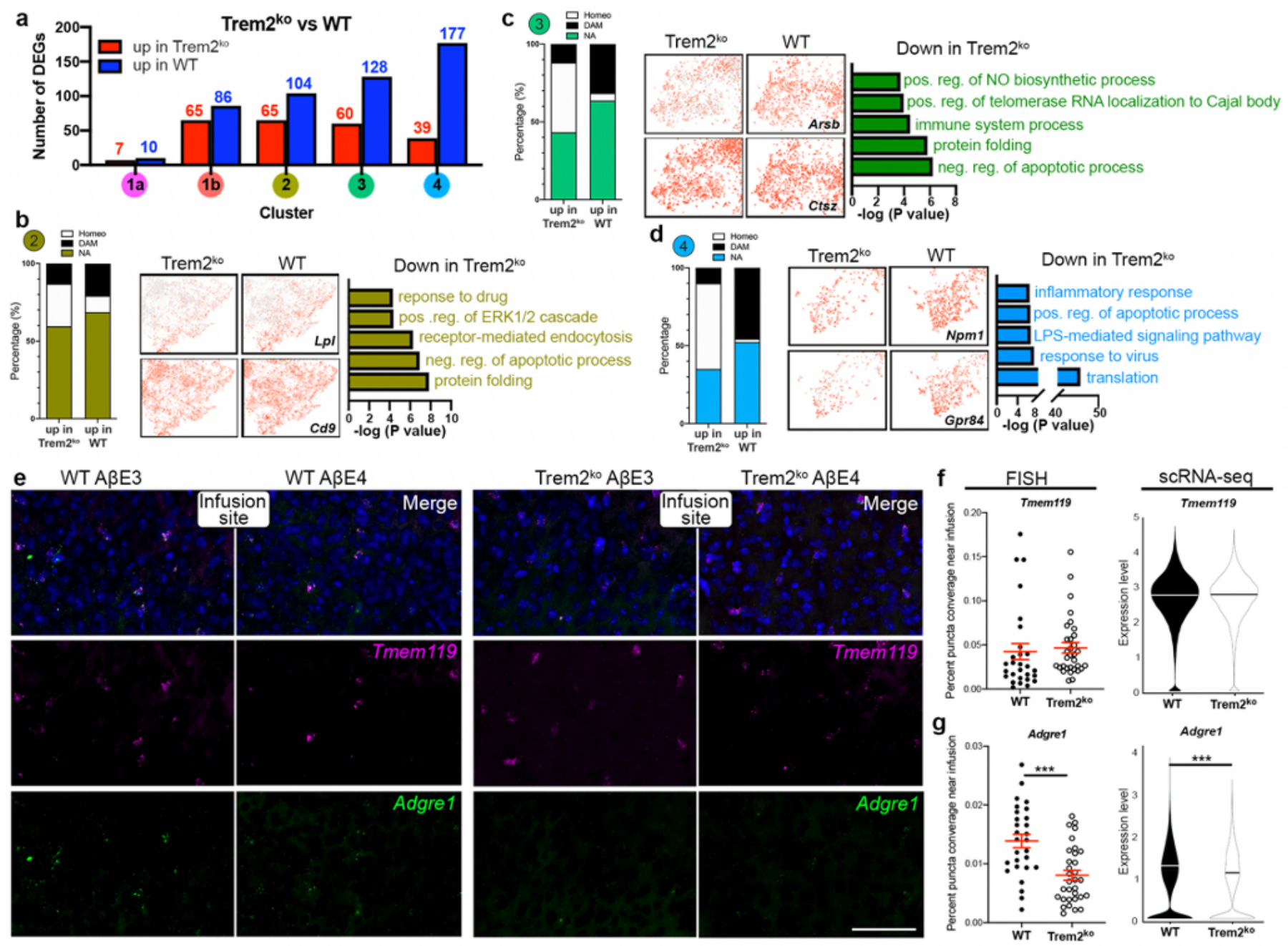

\section{Figure 6}

scRNA-seq identifies activated gene clusters downregulated in Trem2ko mice following $A \beta E 3$ or $A \beta E 4$ infusions. (a) The number of DEGs in Trem2ko compared to WT in each cluster (Bonferroni-corrected pvalue $<0.05$ and natural $\log F C>0.1)$. (b-d) The proportion of homeostatic (Homeo) or DAM genes among DEGs in Trem2ko or WT groups in clusters 2 (b), 3 (c), and 4 (d). Feature plots (Left) showing representative genes and bar plot (Right) showing top 5 GO terms that are significantly down-regulated in Trem2ko compared to WT microglia in the three infusion responsive clusters. (e-g) The expression of Tmem119 and Adgre1 was spatially visualized at $200 \mu \mathrm{m}$ perimeter from the injection site. (e) Representative images of FISH analyses of microglial gene expression near the A $\beta$ infusion site. 
(Tmem119 - pink, Adgre1 - Green, Nuclei - Blue). (f-g) Scatter dot plots showing the area of puncta coverage adjacent to the infusion site along with violin plots of the expression of the same gene in the scRNA-seq dataset for Tmem119 (f), and Adgre1 (g). N = 8 mice per group (equal males and females), with four infusion sites analyzed per animal, red lines represent mean $\pm S E M, \star \star \star ~ p<0.001$ using $t$ test.
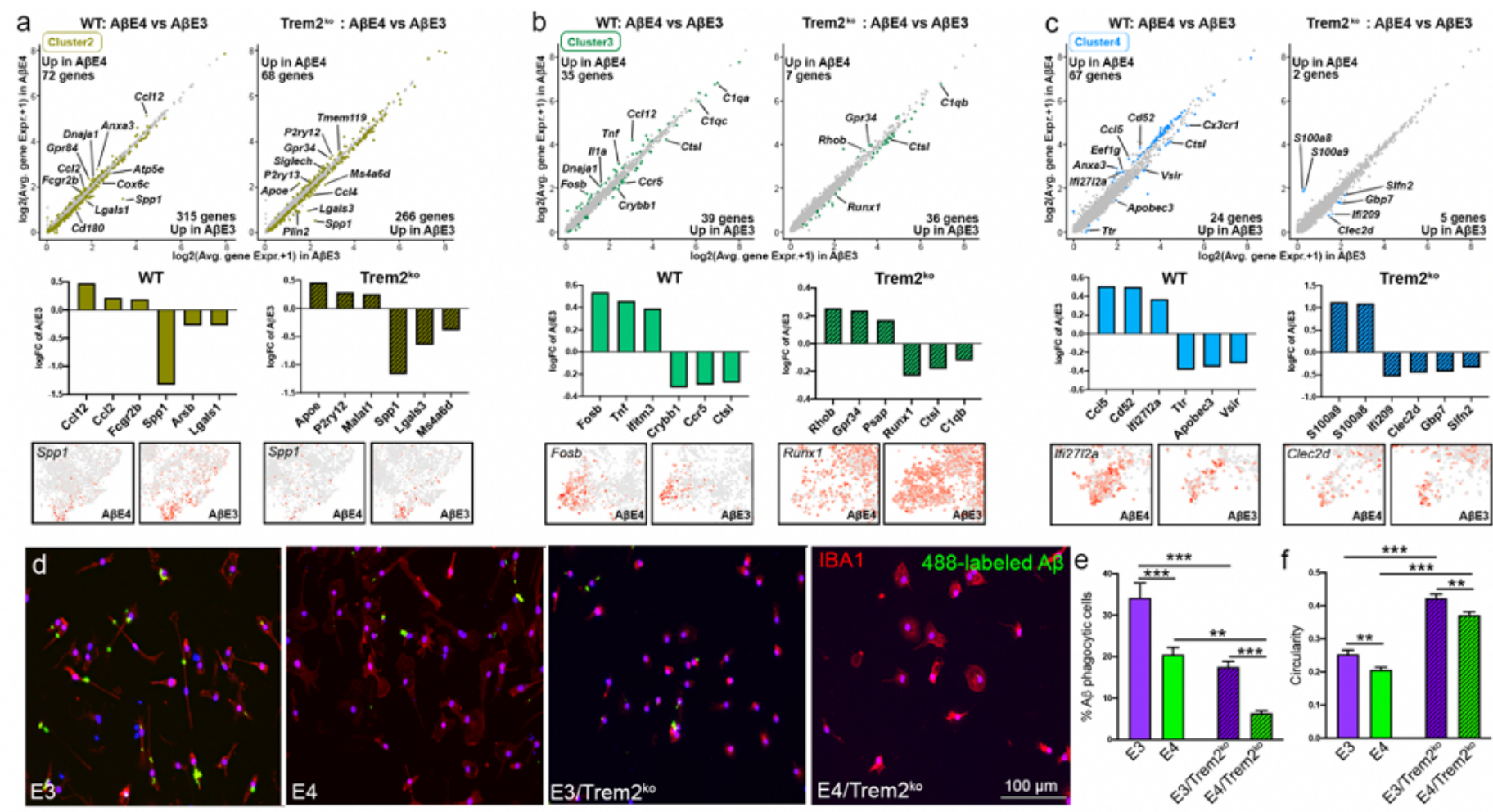

\section{Figure 7}

Trem2 deficiency and APOE isoform affect microglia response to $A \beta(a-c)$ Scatter plots, bar plots and feature plots showing the differentially expressed genes in microglia from WT and Trem2ko mice injected with $A \beta E 4$ or ABE3 complexes for cluster 2 (a), cluster 3 (b), and cluster 4 (c). Differentially expressed genes at $p<0.01$ are colored in each cluster color. The adjusted P-value was performed using Bonferroni

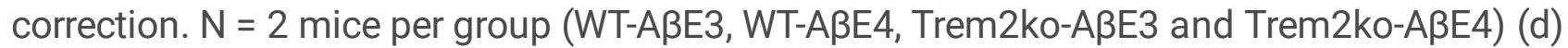
Representative images of microglia from E3, E3/Trem2ko, E4, and E4/Trem2ko mice labeled with IBA1 in red and 488-labeled $A \beta$ in green from three independent cultures in triplicate for each genotype. (e) Bar plot showing the average percentage of A囚-containing microglia for each genotype. (f) Bar plot showing the average circularity of microglia across all images taken for each genotype. Analysis by one-way ANOVA followed by Tukey's multiple comparison test. Bars represent mean $\pm S E M$. * $p<0.05$, ${ }^{\star *} p<0.01$; $\star \star \star ~ p<0.001$, N.S. no significance. 


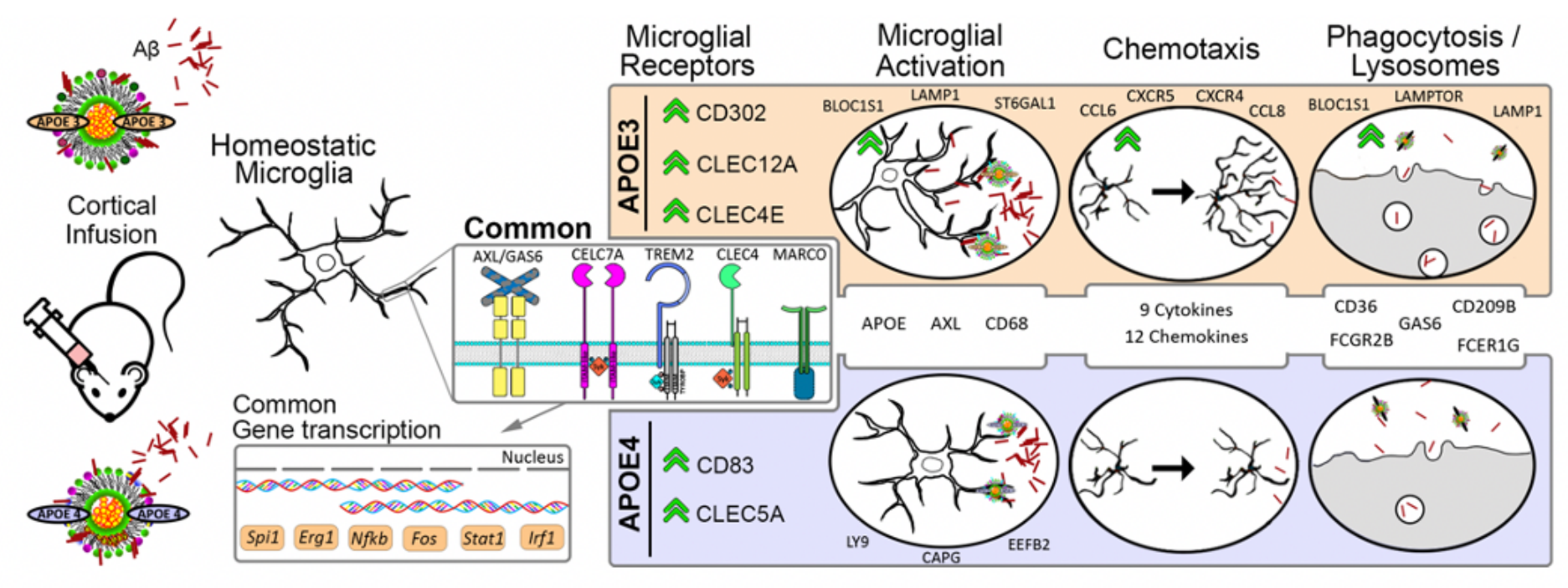

Figure 8

Illustration of the APOE isoform-specific phospholipid composition effects on microglia transcriptome and phenotype in response to A 3 . Shotgun and LC-MS lipidomic analysis of APOE3 and APOE4 astrocyte conditioned media identified isoform-specific differences in phospholipid composition of APOEcontaining lipoproteins. E3 or E4 lipoproteins co-infused with $A \beta$ differentially impacted $A \beta$ binding to microglia receptors (AXL, different CLECs clusters, TREM2, CD83 and others) this in turn affects genes expression of transcription factors (Spi1, Stat1, Nfkb, Irf1) resulting in microglia activation. Active microglia demonstrate characteristic transcriptional profile, increased secretion of cytokines and chemokines thus facilitating chemotaxis and phagocytosis. Green arrows indicate an upregulation of that gene or category compared to the other APOE isoform.

\section{Supplementary Files}

This is a list of supplementary files associated with this preprint. Click to download.

- nrreportingsummaryKoldamova.pdf

- SupplementalFigures.docx 